\title{
Thermodynamics, maximum power, and the dynamics of preferential river flow structures at the continental scale
}

\author{
A. Kleidon ${ }^{1}$, E. Zehe ${ }^{2}$, U. Ehret ${ }^{2}$, and U. Scherer ${ }^{2}$ \\ ${ }^{1}$ Max-Planck Institute for Biogeochemistry, Hans-Knöll-Str. 10, 07745 Jena, Germany \\ ${ }^{2}$ Institute of Water Resources and River Basin Management, Karlsruhe Institute of Technology - KIT, Karlsruhe, Germany
}

Correspondence to: A. Kleidon (akleidon@bgc-jena.mpg.de)

Received: 14 May 2012 - Published in Hydrol. Earth Syst. Sci. Discuss.: 11 June 2012

Revised: 14 December 2012 - Accepted: 22 December 2012 - Published: 22 January 2013

\begin{abstract}
The organization of drainage basins shows some reproducible phenomena, as exemplified by self-similar fractal river network structures and typical scaling laws, and these have been related to energetic optimization principles, such as minimization of stream power, minimum energy expenditure or maximum "access". Here we describe the organization and dynamics of drainage systems using thermodynamics, focusing on the generation, dissipation and transfer of free energy associated with river flow and sediment transport. We argue that the organization of drainage basins reflects the fundamental tendency of natural systems to deplete driving gradients as fast as possible through the maximization of free energy generation, thereby accelerating the dynamics of the system. This effectively results in the maximization of sediment export to deplete topographic gradients as fast as possible and potentially involves large-scale feedbacks to continental uplift. We illustrate this thermodynamic description with a set of three highly simplified models related to water and sediment flow and describe the mechanisms and feedbacks involved in the evolution and dynamics of the associated structures. We close by discussing how this thermodynamic perspective is consistent with previous approaches and the implications that such a thermodynamic description has for the understanding and prediction of subgrid scale organization of drainage systems and preferential flow structures in general.
\end{abstract}

\section{Introduction}

River networks are a prime example of organized structures in nature. The effective rainfall, or runoff, from land does not randomly diffuse through the soil to the ocean, but rather collects in channels that are organized in tree-like structures along topographic gradients. This organization of surface runoff into tree-like structures of river networks is not a peculiar exception, but is persistent and can generally be found in many different regions of the Earth. Hence, it would seem that the evolution and maintenance of these structures of river networks is a reproducible phenomenon that would be the expected outcome of how natural systems organize their flows. The aim of this paper is to understand the basis for why drainage systems organize in this way and relate this to the fundamental thermodynamic trend in nature to dissipate gradients as fast as possible. Such a basis will likely help us to better understand the central question of hydrology regarding the partitioning of precipitation into evaporation and runoff from first principles.

\subsection{River systems and organizational principles}

Several approaches have tried to understand this form of organization from basic organization principles that involve different forms of energetic optimization (see, e.g. the review by Phillips, 2010 and Paik and Kumar, 2010) or from stability analysis of the conservation of sediment and water and transport laws (e.g. Kirkby, 1971; Smith and Bretherton, 1972). While the latter studies also provide explanations for the evolution of spatial structures in river basins, these studies do not consider changes in energy specifically. We focus here on those studies that deal with principles that explicitly treat conversions of energy, as these are most closely related to thermodynamics and the second law, and thus should have the greatest potential for formulating organizational principles in the most general terms. 
In terms of energetic principles, Woldenberg (1969) showed that basic scaling relationships of river basins can be derived from optimality assumptions regarding stream power. Similarly, Howard (1990) described optimal drainage networks from the perspectives that these minimize the total stream power, while Rodriguez-Iturbe et al. (1992a,b) and Rinaldo et al. (1992) used the assumption of "minimum energy expenditure" (also Leopold and Langbein, 1962; Rodriguez-Iturbe and Rinaldo, 1997) and were able to reproduce the observed, fractal characteristics of river networks. Similar arguments were made by Bejan (1997) in the context of a "constructal law", which states that the evolution of river networks should follow the trend to maximize "access" (the meaning of "access", however, is ambiguous and difficult to quantify). Likewise, West et al. (1997) showed that the assumption of minimizing frictional dissipation in three dimensional networks yields scaling characteristics in trees and living organisms that are consistent with observations.

Related to these energetic minimization principles are principles that seem to state exactly the opposite: that systems organize to maximize power, dissipation or, more generally, entropy production. These three aspects are closely related. While power, the rate at which work is performed through time, describes the generation of free energy, this free energy is dissipated into heat in a steady state, resulting in entropy production. This maximization is also related to minimization. When frictional dissipation of a moving fluid is minimized, its ability to transport matter along a gradient is maximized. This aspect is further explored in more detail in this manuscript for the case of river networks as well as their surrounding hillslopes. Hence, the maximization of any of these aspects in steady state yields roughly the same result, namely, that driving gradients that yield the power to drive the dynamics are dissipated as fast as possible. The maximum power principle was originally formulated in electrical engineering in the 19th century, and found repeated considerations in biology (Lotka, 1922a,b), ecology (Odum, 1969, 1988) and Earth system science (e.g. Kleidon, 2010a). Closely related but developed independently, the proposed principle of Maximum Entropy Production (MEP) was first formulated in atmospheric sciences by Paltridge $(1975,1979)$ and has recently gained attention, e.g. in attempting to derive it theoretically from statistical physics and information theory (Dewar, 2005, 2010), in applying it to a variety of environmental systems (Kleidon et al., 2010; Kleidon, 2010b) and to land surface hydrology in particular (e.g. Wang and Bras, 2011; Kleidon and Schymanski, 2008). A recent example of the application of maximum dissipation to preferential water flow in soils is given in Zehe et al. (2010).

In this paper, we use a thermodynamic perspective of the whole continental system to show that these proposed optimality principles are not contrary to each other, but all reflect the overall trend in Earth system functioning to deplete driving gradients as fast as possible. The term "as fast as possible" is non-trivial and is fundamentally constrained by the conservation laws of energy, mass, and momentum. Applied to river network structures, this general trend translates into the hypothesis that these network structures form because they represent the means to deplete the topographic gradients at the fastest possible rate. This might appear counterintuitive at first sight. It would seem that the second law of thermodynamics would imply that gradients and thus spatial organization are depleted, and not created. As we will see below, it is through a "detour" of structure formation that the overall dynamics to deplete gradients are accelerated and hence the presence of structure can be interpreted as the result of the second law of thermodynamics in a broader sense. To evaluate this hypothesis, we need to understand the energetic limits to sediment transport, but we also need to take a broader view of what is driving continental dynamics and topographic gradients in the first place as these set the flexible boundary conditions for river flow and its organization.

\subsection{River systems in the broader, continental context}

To understand the depletion of topographic gradients in relation to changes in energy and the second law, we need to look at the broader context of the processes that shape topographic gradients. This context involves the dynamics of the continental crust as illustrated in Fig. 1 in a highly simplified way. This figure shows the dynamics of topographic gradients on land in terms of three steps from an initial state of local, isostatic equilibrium of continental crust to a state of global, "stratigraphic" equilibrium in which continental crust is uniformly distributed over the planet. This trend from Fig. 1a to $\mathrm{c}$ is reflected in an energetic trend of decreased potential energy. In this idealized setup, we make the simplifying assumption that there is no tectonic activity that would act to form and concentrate continental crust and thus maintain the generation of continents.

To relate the trend to energetic changes, we note that the energy that describes the system consists of the potential energies of continental and oceanic crust. Continental crust has a lower density than oceanic crust. At our starting point, Fig. 1a, the two masses are in a state of local, isostatic equilibrium. This state is associated with no uplift or subsidence of continental crust, since the buoyancy force due to the difference in densities is balanced by gravity. This local state is associated with a horizontal gradient in topography, here represented by the difference $\Delta z=\Delta z_{\mathrm{c}, 1}-\Delta z_{\mathrm{o}, 1}$, which measures the tops of the two types of crust to a reference depth.

The lowest value of potential energy in the system would be achieved in a state of "global equilibrium", in which the material of the continental crust is uniformly spread out over the whole surface of the Earth, as shown in Fig. 1c. The associated reduction in potential energy is shown mathematically in the Appendix. In this state, the potential energy of the oceanic crust and the upper mantle would overall be lowered to an elevation below $\Delta z_{\mathrm{o}, \mathrm{g}}$, while the potential energy 
a. local, isostatic equilibrium

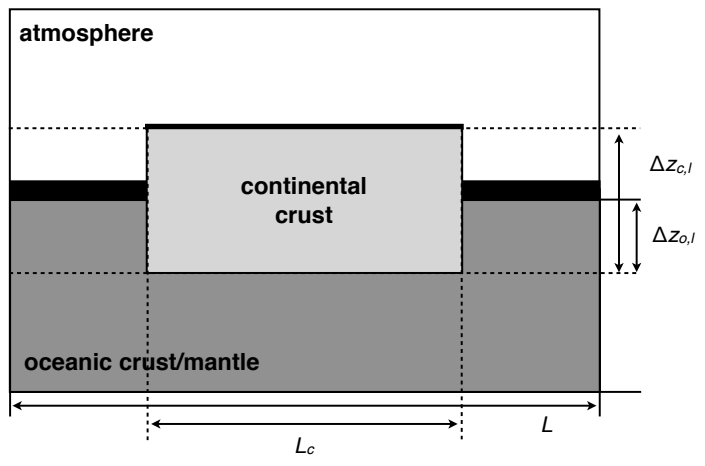

b. local disequilibrium by sediment transport

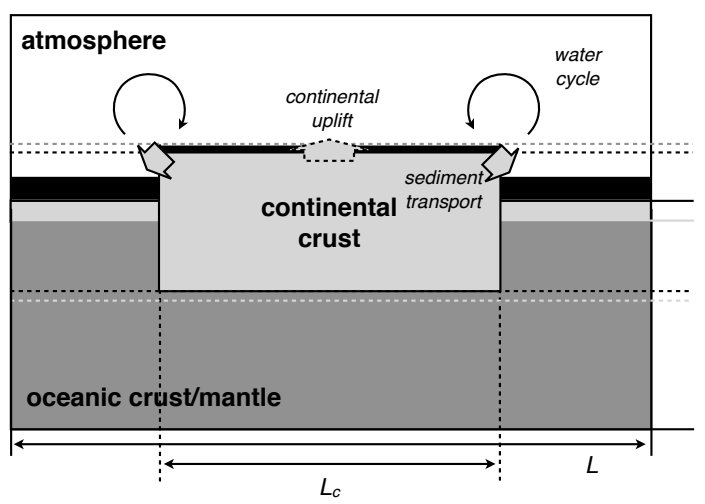

c. global, stratigraphic equilibrium

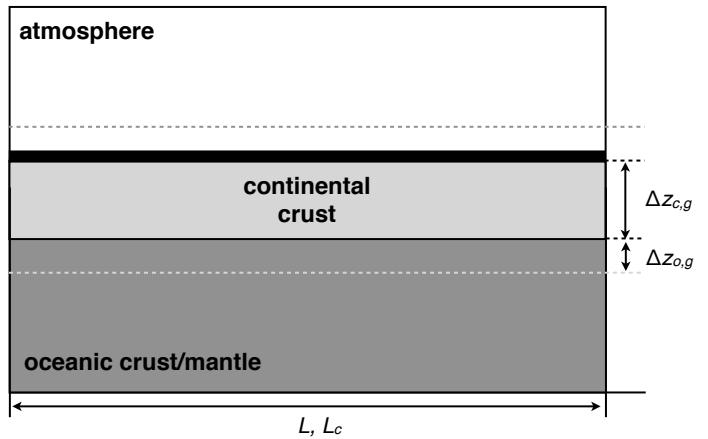

Fig. 1. Highly simplified diagram to illustrate how continental crust evolves from (a) a state of local, isostatic equilibrium through (b) a state with sediment transport to (c) a state of global, stratigraphic equilibrium. Sediment transport provides the means to efficiently transport continental crust along topographic gradients in the horizontal and thereby minimizes the potential energy within the system (see Appendix for details). The ocean is shown in black and plays a critical role here as the driver of the hydrologic cycle (thin arrows), which in turn provides a substantial power source to accelerate sediment transport. Plate tectonics is excluded for simplicity. The symbols in the figures are used in the Appendix to quantify this direction towards minimizing the potential energy associated with oceanic and continental crust. of the continental crust would be lowered to an elevation below $\Delta z_{\mathrm{c}, \mathrm{g}}$.

The critical point relating to the role of river networks is that getting from step (a) to (c) without fluvial transport of sediments is extremely slow. With the work done by runoff and river flow in organized network structures on sediment transport, the depletion of the driving gradient $\Delta z$ is, overall, substantially enhanced, possibly to the fastest possible rate allowed by the system setting. Hence, our hypothesis relates to step (b) shown in Fig. 1b. To evaluate this hypothesis, we need to consider the response of continental uplift to the erosion of topographic gradients by sediment transport.

\subsection{Structure of the paper}

In the following, we first provide a brief overview of thermodynamics to provide the context of a thermodynamic description of the Earth system in Sect. 2. We then formulate drainage systems as thermodynamic systems and describe their dynamics in terms of conversions of energy of different forms. We then set up three simple models to demonstrate the means by which drainage basins act to maximize sediment transport and thereby the depletion of geopotential gradients of continental crust. These examples are kept extremely simple to show that such maximum states exist and what it needs to evolve to these maximum states. In Sect. 5 we then explore why the evolution and dynamics of structure formation associated with river networks should be directed towards achieving these maximum power states. In Sect. 6 we characterize these dynamics in terms of different time scales that are based on rates of free energy generation and gradient depletion and the associated feedbacks that shape the dynamics. In the discussion we then relate our results to previous work on river networks, in particular to proposed energy minimization principles, and more generally to thermodynamics and optimality and explore the implications of these results. We close with a brief summary and conclusion.

\section{Brief overview of the thermodynamics of Earth system processes}

Thermodynamics is a fundamental theory of physics that deals with the general rules and limits for transforming energy of different types. It is commonly applied to conversions that involve heat, and to systems with fixed boundary conditions, such as a heat engine. The scope of thermodynamics is, however, much wider. In the following overview, we sketch out the common basis to describe a system in terms of exchanges of energy of different forms and how the first and second law of thermodynamics provide the limits of conversion rates from one form of energy into another. We then describe how thermodynamics provides the basis to describe the dynamics of systems in the context of Earth system functioning at large. 
Table 1. Different forms of energy relevant for the description of drainage basin dynamics and their thermodynamic description as pairs of conjugate variables, one extensive variable that depends on the size of the system, and one intensive variable that is independent of the size of the system.

\begin{tabular}{lllll}
\hline form of energy & $\begin{array}{l}\text { extensive } \\
\text { variable }\end{array}$ & $\begin{array}{l}\text { intensive variable } \\
\text { variable }\end{array}$ & $\begin{array}{l}\text { expression } \\
\text { for work }\end{array}$ & $\begin{array}{l}\text { associated fluxes } \\
\text { and conservation laws }\end{array}$ \\
\hline thermal & $\begin{array}{l}\text { entropy } \\
\text { temperature }\end{array}$ & $\begin{array}{l}\mathrm{d} W=\mathrm{d}(S T) \\
\text { kinetic }\end{array}$ & $\begin{array}{l}\text { momentum } \\
p=m v\end{array}$ & $\begin{array}{l}c_{\mathrm{p}} \rho \mathrm{d} T / \mathrm{d} t=\Sigma J_{\mathrm{h}} \\
\text { (heat balance) }\end{array}$ \\
\hline $\begin{array}{l}\text { potential } \\
\text { (or gravitational) }\end{array}$ & $m$ & $\mathrm{~d} W=\mathrm{d}(p v)$ & $\begin{array}{l}\mathrm{d} p / d \mathrm{t}=\Sigma F \\
\text { (momentum balance) }\end{array}$ \\
& $m$ & $\begin{array}{l}\text { geopotential } \\
\text { (or gravitational } \\
\text { potential) } \\
g z\end{array}$ & $\mathrm{~d} W=\mathrm{d}(m g z)$ & $\begin{array}{l}\mathrm{d} m / \mathrm{d} t=\Sigma J_{\mathrm{m}} \\
\text { (mass balance) }\end{array}$ \\
\hline
\end{tabular}

We start with the general description of a system in terms of its various contributions to the total energy $U$ of the system. The different forms of energy can be described in terms of sets of conjugate variables, consisting each of an intensive variable that is independent of the size of the system, such as temperature, pressure, charge, surface tension or geopotential, and an extensive variable, which depends on the size of the system, such as entropy, volume, voltage, surface area or mass. A brief overview of these sets of variables and the related forms of energy relevant here is summarized in Table 1 , while an overview of the thermodynamic terminology is provided in Table 2 .

The formulation of the dynamics of a system in terms of the conjugate variables and associated forms of energy set the basis for applying the first and second law of thermodynamics to the dynamics. The first law of thermodynamics essentially states the conservation of energy, i.e. it states that the sum of all changes of energy within the system balances the energy exchanges with the surroundings. Traditionally, the first law is expressed as the change in total energy $\mathrm{d} U$ of the system being balanced by external heating $\mathrm{d} Q$ and the work done by the system $\mathrm{d} W$ :

$\mathrm{d} U=\mathrm{d} Q-\mathrm{d} W$.

When we take a broader view of the total energy of the system, then $\mathrm{d} W$ is not removed from the system, but rather converted into another form of energy. For instance, when a small amount of work $\mathrm{d} W$ is being performed from differential heat to generate motion, kinetic energy is increased by $\mathrm{d} W$ at the expense of heat, which is reduced by $-\mathrm{d} W$. During this conversion process, the total energy of the system remains constant, $\mathrm{d} U=0$, and it is merely the form of energy that is being altered. When we include these forms of energy as contributions to the total energy of the system, then the first law limits the energy conversions within the system, and the $\mathrm{d} W$ term represents the conversion of heat to some other form of energy. More specifically, the $\mathrm{d} W$ term represents the work done to create a gradient in another variable under conservation of mass, momentum and other conservation laws. For instance, when motion is generated (i.e. work is performed to accelerate mass), this corresponds to the generation of a velocity gradient at the expense of exploiting another gradient (e.g. heating or geopotential). When work is performed to lift mass, it corresponds to the generation of a gradient in the geopotential, again, at the expense of exploiting another gradient (e.g. a velocity gradient). Hence, the dynamics within the system is all about converting gradients associated with one form of energy into gradients of another form of energy. In a broader sense, the first law tells us to do the proper accounting of the build-up and depletion of gradients of different types. These gradients allow work to be derived from them, so these gradients are associated with free energy, i.e. energy that is able to perform work. Note that sometimes this is referred to as "exergy", or specific forms of free energy are used (e.g. Gibbs free energy, Helmholtz free energy). In the following, we will refer to the term "free energy" in a general way as a gradient in a variable associated with a certain form of energy that can be used to generate another gradient. We will refer to the generation term $\mathrm{d} W / \mathrm{d} t=P$ as the power associated with this conversion. In this context, a broader interpretation of the first law tells us that the total of all energy conversions between different forms of energy within a system need to balance the energy exchanges with the surroundings.

The second law of thermodynamics states that the entropy of an isolated system can only increase. When this law is extended to non-isolated systems that exchange energy and/or mass, it takes the form of a constraint for the budget of the system's entropy $S$ :

$\mathrm{d} S / \mathrm{d} t=\sigma+\sum_{i} J_{\mathrm{s}, i}$,

where $\sigma \geq 0$ is the entropy produced within the system by irreversible processes, and $\sum_{i} J_{\mathrm{s}, i}$ is the sum of all entropy exchange fluxes with the surroundings associated with energyand mass exchange. By constraining $\sigma$ to values greater or 
Table 2. Overview of the different thermodynamic terms used here, their brief definitions and their relevance to hydrologic processes.

\begin{tabular}{|c|c|c|}
\hline term & description & examples used here \\
\hline conjugate variables & $\begin{array}{l}\text { A set of two variables for which the product describes a } \\
\text { form of energy. The pair is formed by one intensive and } \\
\text { one extensive variable. }\end{array}$ & see Table 1 \\
\hline extensive variable & a variable that depends on the size of the system & stocks of water (soil, river, water vapor), momentum of flow \\
\hline intensive variable & a variable that does not depend on the size of the system & geopotential (or gravitational potential), flow velocity \\
\hline heat & $\begin{array}{l}\text { a specific form of energy measured by temperature } \\
\text { (better term: thermal energy) }\end{array}$ & soil heat storage \\
\hline work & $\begin{array}{l}\text { the conversion of one form of energy into another; } \\
\text { mechanical definition: the exertion of a force over a } \\
\text { distance }\end{array}$ & acceleration or lifting of water and sediment \\
\hline entropy & $\begin{array}{l}\text { unavailability of a system's thermal energy for } \\
\text { conversion into mechanical work. }\end{array}$ & $\begin{array}{l}\text { thermal energy is only considered in this manuscript as } \\
\text { the end result of dissipative processes }\end{array}$ \\
\hline free energy & the capacity of a form of energy to perform work & potential energy of surface water, kinetic energy of river flow \\
\hline disequilibrium & $\begin{array}{l}\text { the presence of a gradient in conjugate variables, } \\
\text { associated with the presence of free energy of some form }\end{array}$ & gradients in geopotential, velocity \\
\hline power & $\begin{array}{l}\text { the generation rate of free energy of a particular process } \\
\text { at the expense (i.e. depletion) of another gradient }\end{array}$ & $\begin{array}{l}\text { generation rate of kinetic energy of stream flow resulting } \\
\text { from the depletion of potential energy of water }\end{array}$ \\
\hline $\begin{array}{l}\text { generation rate of free } \\
\text { energy }\end{array}$ & $\begin{array}{l}\text { rate of increase in free energy of a particular form (same } \\
\text { as power) }\end{array}$ & $\begin{array}{l}\text { generation rate of potential and kinetic energy of water } \\
\text { and sediment }\end{array}$ \\
\hline transfer & $\begin{array}{l}\text { the increase of free energy of one form due to the } \\
\text { depletion of another form }\end{array}$ & $\begin{array}{l}\text { free energy transfer from river flow to sediment } \\
\text { transport }\end{array}$ \\
\hline import of free energy & transport of free energy across the system boundary & import of geopotential energy through precipitation \\
\hline dissipation & $\begin{array}{l}\text { the depletion of free energy by an irreversible process } \\
\text { into heat }\end{array}$ & frictional dissipation in fluid flow \\
\hline depletion rate & $\begin{array}{l}\text { the reduction of free energy either by dissipation or by } \\
\text { conversion into another form }\end{array}$ & $\begin{array}{l}\text { water flow and sediment export deplete gradients of } \\
\text { potential energy }\end{array}$ \\
\hline irreversibility & $\begin{array}{l}\text { not able to be undone without the performance of work, } \\
\text { i.e. processes that dissipate free energy }\end{array}$ & frictional dissipation in fluid flow \\
\hline
\end{tabular}

equal to zero, the second law provides the direction into which processes evolve. This law is reflected in the spontaneous depletion of gradients. For instance, heating gradients are dissipated by heat conduction, while velocity gradients are dissipated by friction. Hence, a broader interpretation of the second law implies that natural processes are directed such that they deplete their driving gradients.

To obtain the limits to how much mechanical work can be extracted from a heating source, as for instance is the case for a classical heat engine, the combination of the first and second law result in the well-known Carnot limit. To outline the derivation of this limit, we consider a fixed influx of heat into a system $J_{\mathrm{h}, \text { in }}$ from a hot reservoir with fixed temperature $T_{\mathrm{h}}$ and a heat flux $J_{\mathrm{h} \text {,out }}$ from the system to a cold sink with fixed temperature $T_{\mathrm{c}}$. The rate at which power can be extracted is given by the first law (noting that $\mathrm{d} Q / \mathrm{d} t=J_{\mathrm{h} \text {,in }}-J_{\mathrm{h} \text {,out }}$ and $P=\mathrm{d} W / \mathrm{d} t$, both being in units of Watt $\mathrm{W}$, or $\mathrm{W} \mathrm{m}^{-2}$ while heat $Q$ has the unit of $\mathrm{J}$ or $\mathrm{J} \mathrm{m}^{-2}$ ):
$J_{\mathrm{h}, \text { in }}-J_{\mathrm{h}, \text { out }}=P$.

When we assume that no entropy is produced within the system (i.e. $\sigma=0$ ), which is rather optimistic and serves merely to establish the upper limit for $P$, we can then derive an expression of the maximum power $P_{\max }$ that can be extracted from these heat fluxes by noting that the net entropy exchange of the system cannot become negative to fulfill the second law:

$\sum_{i} J_{\mathrm{s}, i}=J_{\mathrm{h}, \text { out }} / T_{\mathrm{c}}-J_{\mathrm{h}, \text { in }} / T_{\mathrm{h}} \geq 0$

using the expression of $\mathrm{d} S=\mathrm{d} Q / T$ for expressing the entropy of a heat flux. The entropy budget can be rearranged to yield an expression for $J_{\mathrm{h} \text {,out }}\left(\geq J_{\mathrm{h} \text {,in }} T_{\mathrm{c}} / T_{\mathrm{h}}\right)$ such that the second law is fulfilled. Taken together with the first law, this yields the well-known expression for the Carnot limit (see comment by Kleidon et al., 2012b for a derivation of this equation): 
$P \leq P_{\max }=J_{\mathrm{h}, \text { in }}\left(T_{\mathrm{h}}-T_{\mathrm{c}}\right) / T_{\mathrm{h}}$.

When we relax the assumptions in this derivation and allow for (a) other processes to deplete the temperature gradient (e.g. diffusion or radiative exchange) so that entropy is produced within the system and (b) the temperature gradient is affected by the generated power (e.g. by the convective heat flux that is associated with the resulting motion), then one can obtain a very similar expression for a maximum power limit that is reduced by a factor of 4 due to the decrease in the temperature gradient and due to a competing dissipative process (Kleidon, 2012). We can generalize this maximum power limit to apply to practically all forms of energy conversions, particularly to the ones involved in river flow and sediment transport. We will describe the application to drainage basins in Sect. 3 .

When we now consider the dynamics of a system in the context of the functioning of the Earth system at large, we first note that free energy plays a central role in describing the interactions of the system with the Earth (Fig. 2). First, free energy is ultimately derived and transformed from the two planetary forcings of solar radiation and interior cooling through a sequence of energy conversions. Thermodynamics, as outlined above, is the basis to account for these conversions and inherent limits. The surface water at some elevation above sea level (a.s.l.) has the potential energy that can be converted to the kinetic energy associated with runoff. This potential energy is generated by the atmospheric cycling of water. The cycling of water, in turn, is driven by atmospheric motion, which is driven by the differential heating associated with solar radiation. Likewise, the sediment that is eroded by water flow gained its potential energy through lifting of continental crust, which is related to the motion of plates and the mantle, which is ultimately driven by heating gradients between the Earth's interior and the surface. It is only through this broader perspective that we can fully account for the origin and the limits of free energy transfer from the primary drivers to the dynamics of a drainage basin.

In the following section we will nevertheless focus on the forms of energy that are directly involved in the generation of river flow and sediment transport, with the larger-scale forcing taken as inputs of the associated forms of free energy.

\section{Drainage basins as thermodynamic systems}

We consider continental drainage basins as open thermodynamic systems that exchange mass and energy with their surroundings (Fig. 3). Incoming mass fluxes at elevations a.s.l. add geopotential free energy to the system. The following description does not use thermodynamic analogies, as it was done, for instance, by Leopold and Langbein (1962) who viewed a river as a set of heat engines with water flow being an analogy to a heat flow along a temperature gradient. Heat is not a direct driver of the dynamics of river flow, but rather gradients in geopotential. We take thermodynamics as our starting point for the description of energy transfers in drainage basins as it provides the framework to describe energy and energy conversions in general. The labeling convention for variable names as well as an overview of variables used in the following is summarized in Table 3.

\subsection{Definition of drainage systems as thermodynamic systems}

The starting point for a thermodynamic description is the total energy $U$ of the drainage system. In the simple illustration used here, the relevant contributions to $U$ are the geopotential energy of surface water (index " $w$ ") and continental mass (index "s"), the kinetic energy of water and sediment flow, as well as the dissipative heating sink term. Hence, changes in total energy $\mathrm{d} U$ are expressed as

$\mathrm{d} U=\mathrm{d}\left(m_{\mathrm{w}} \phi_{\mathrm{w}}\right)+\mathrm{d}\left(m_{\mathrm{s}} \phi_{\mathrm{s}}\right)+\mathrm{d}\left(p_{\mathrm{w}} v_{\mathrm{w}}\right)+\mathrm{d}\left(p_{\mathrm{s}} v_{\mathrm{s}}\right)+\mathrm{d}(T S)$,

where $m_{\mathrm{w}}$ and $m_{\mathrm{S}}$ are the mass of surface water and continental crust within the system at certain geopotentials $\phi_{\mathrm{w}}$ and $\phi_{\mathrm{s}}$, respectively, $p_{\mathrm{w}}$ and $p_{\mathrm{s}}$ the momentum associated with water and suspended sediment with velocities $v_{\mathrm{w}}$ and $v_{\mathrm{s}}$, and $T$ and $S$ being the temperature and entropy within the system. For simplicity, we do not consider the forms of energy (particularly, binding energies) and the associated processes involved in the conversion of rock into sediment (i.e. physical and chemical weathering or the wetting and drying of soils). We assume that the continental mass already consists of loose sediment particles and thus only consider the motion of continental mass suspended in water flow in form of sediments. Furthermore, we neglect bedload and dissolved transport as well as debris flows. While these processes play important roles in transporting continental crust to the ocean, we focus here only on sediment transport. This focus is justified because we aim to understand the role of river network structures, and these structures are formed by the redistribution of sediment mostly by fluvial processes. Hence, at a minimum, we need to consider the potential and kinetic energy of water and sediments.

\subsection{Thermodynamic equilibrium}

To identify the state of thermodynamic equilibrium for the forms of energy considered in Eq. (6) in the catchment system, we exclude exchange fluxes from our consideration, so that the total energy of the system as well as its mass and momentum are conserved. For simplicity, we lump kinetic and potential energy associated with water and sediment into a single variable $A$ that expresses the non-heat related forms of energy. When we then assume that the system is approximately isothermal so that changes in $T$ can be neglected (after all, the dynamics within the system do not result in substantial heating within the system), we can then write Eq. (6) as: 
Table 3. Overview of the parameter and variable names used in the models. The variables follow a terminology in which all fluxes of a property are described by $J$, the generation of free energy of some form from another form is described by $P$, the dissipation of free energy into heat by $D$, and forces by $F$. The subscript index refers to the substance (w: water, s: sediment), while the superscript refers to the type of flux (no superscript: mass; $p$ : momentum; ke: kinetic energy; pe: potential energy).

\begin{tabular}{|c|c|c|}
\hline symbol & description & units \\
\hline$m_{\mathrm{W}}, m_{\mathrm{s}}$ & mass of water and sediments & $\mathrm{kg}$ \\
\hline$\phi$ & geopotential (or gravitational potential) & $\mathrm{m}^{2} \mathrm{~s}^{-2}$ \\
\hline$p_{\mathrm{W}}, p_{\mathrm{s}}$ & momentum associated with water and sediment flow & $\mathrm{kg} \mathrm{ms} \mathrm{s}^{-1}$ \\
\hline$v$ & velocity of water and sediment flow (assumed to be equal) & $\mathrm{ms}^{-1}$ \\
\hline$J_{\mathrm{w}, \text { in }}$ & effective precipitation (import of water into the system) & $\mathrm{kg} \mathrm{s}^{-1}$ \\
\hline$J_{\mathrm{W}, \text { out }}$ & river discharge (export of water from the system) & $\mathrm{kg} \mathrm{s}^{-1}$ \\
\hline$J_{\mathrm{s}, \text { in }}$ & uplift of continental mass (import of sediment into the system) & $\mathrm{kg} \mathrm{s}^{-1}$ \\
\hline$J_{\mathrm{S}, \text { out }}$ & sediment export (export of sediment from the system) & $\mathrm{kg} \mathrm{s}^{-1}$ \\
\hline$F_{\mathrm{W}, \mathrm{acc}}, F_{\mathrm{S}, \mathrm{acc}}$ & $\begin{array}{l}\text { accelerating force for water and sediment flow due to gravity (transfer of } \\
\text { geopotential to momentum) }\end{array}$ & $\mathrm{kg} \mathrm{ms}^{-2}$ \\
\hline$F_{\mathrm{W}, \mathrm{d}}, F_{\mathrm{s}, \mathrm{d}}$ & $\begin{array}{l}\text { drag force on water and sediment flow (momentum transfer from flow to surface at } \\
\text { rest) }\end{array}$ & $\mathrm{kg} \mathrm{ms} \mathrm{s}^{-2}$ \\
\hline$F_{\mathrm{w}, \mathrm{s}}$ & $\begin{array}{l}\text { drag force on water flow that detaches sediment (momentum transfer from water } \\
\text { flow to sediment) }\end{array}$ & $\mathrm{kg} \mathrm{ms}^{-2}$ \\
\hline$F_{\mathrm{W}, \text { crit }}$ & threshold drag needed to detach sediments & $\mathrm{kg} \mathrm{ms}^{-2}$ \\
\hline$J_{\mathrm{w}, \text { out }}^{\mathrm{p}}, J_{\mathrm{s}, \text { out }}^{\mathrm{p}}$ & momentum export associated with water and sediment flow & $\mathrm{kg} \mathrm{ms}^{-2}$ \\
\hline$J_{\mathrm{w}, \text { in }}^{\mathrm{pe}}, J_{\mathrm{s}, \text { in }}^{\mathrm{pe}}$ & import of potential energy by precipitation and uplift & $\mathrm{W}$ \\
\hline$J_{\mathrm{w}, \text { out }}^{\mathrm{pe}}, J_{\mathrm{s}, \text { out }}^{\mathrm{pe}}$ & export of potential energy by runoff and sediment export & $\mathrm{W}$ \\
\hline$J_{\mathrm{w}, \text { out }}^{\mathrm{ke}}, J_{\mathrm{s}, \text { out }}^{\mathrm{ke}}$ & export of kinetic energy by runoff and sediment export & $\mathrm{W}$ \\
\hline$P_{\mathrm{W}}, P_{\mathrm{S}}$ & $\begin{array}{l}\text { generation rate of kinetic energy from potential energy associated with runoff and } \\
\text { sediments }\end{array}$ & $\mathrm{W}$ \\
\hline$D_{\mathrm{w}}, D_{\mathrm{s}}$ & dissipation of kinetic energy associated with runoff and sediment transport & W \\
\hline$P_{\mathrm{w}, \mathrm{s}}$ & free energy transfer rate from water flow to detach and lift sediments & W \\
\hline$N_{\mathrm{d}}, N_{\mathrm{s}}$ & $\begin{array}{l}\text { dimensionless numbers to express the ratio of drag force to geopotential gradient } \\
\text { and settling of sediments to export }\end{array}$ & \\
\hline$f$ & fraction of suspended sediments that is exported & \\
\hline$d_{\mathrm{c}}$ & mean distance to channel & $\mathrm{m}$ \\
\hline$r_{\mathrm{c}}$ & hydraulic radius & $\mathrm{m}$ \\
\hline$N$ & number of drainage channels & \\
\hline$k_{\text {up }}$ & coefficient describing uplift rate & $\mathrm{kg} \mathrm{s} \mathrm{m}^{-1}$ \\
\hline$D \phi$ & measure for disequilibrium associated with structure & $\mathrm{J} \mathrm{kg}^{-1} \mathrm{~m}^{-1}$ \\
\hline$A$ & area & $\mathrm{m}^{2}$ \\
\hline$g$ & gravitational acceleration & $\mathrm{ms} \mathrm{s}^{-2}$ \\
\hline$L$ & horizontal dimension & $\mathrm{m}$ \\
\hline$\Delta z$ & difference in height & $\mathrm{m}$ \\
\hline$\alpha$ & slope & $\circ$ \\
\hline$\mu$ & material property converting the work done on sediment detachment into a mass flux & $\mathrm{kg} \mathrm{J}^{-1}$ \\
\hline$\rho$ & density & $\mathrm{kg} \mathrm{m}^{-3}$ \\
\hline$\tau$ & time scale & $\mathrm{s}$ \\
\hline
\end{tabular}




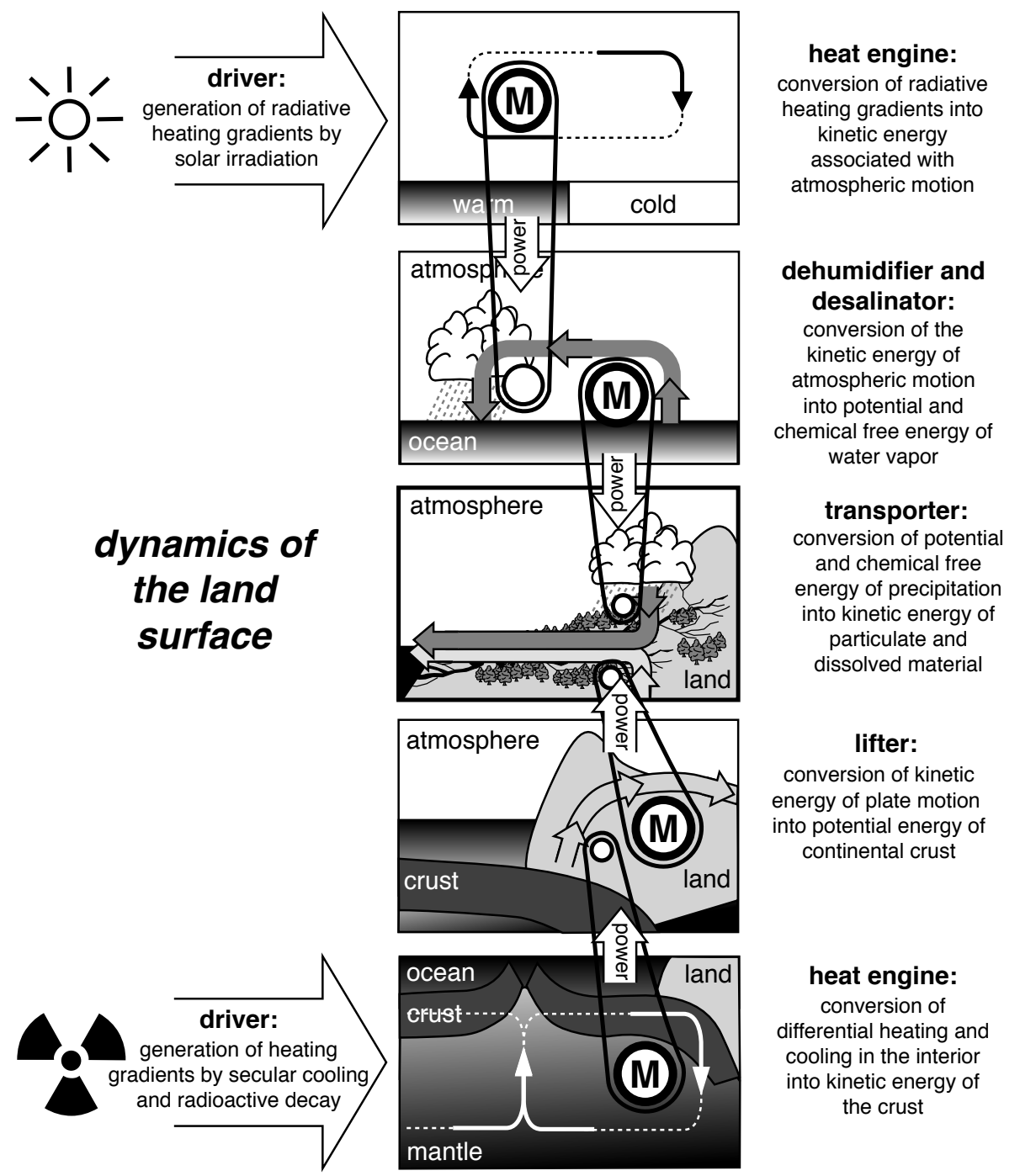

Fig. 2. Schematic diagram illustrating the paths of how free energy is generated and transferred from heating gradients to drive the shaping of drainage systems by geologic and hydrospheric processes. The upper part of the diagram shows how radiative heating gradients fuel the atmospheric heat engine, which in turn acts to dehumidify and desalinate ocean water, which then provides the precipitation input to drive sediment transport. The lower part of the diagram shows how heating gradients in the interior result in plate tectonics and continental uplift, which in turn maintains the topographic gradients for continental river flow. The symbol " $M$ " represents an engine that converts the displayed gradient into power. After Kleidon et al. (2012a).

$\mathrm{d} U=0=\mathrm{d} A+T \mathrm{~d} S$.

Rearranging this to express changes in the entropy of the system yields

$\mathrm{d} S=-\mathrm{d} A / T$.

In other words, the second law of thermodynamics tells us that the dynamics within the system are directed to minimize the kinetic and potential energies of water and sediments.

\subsection{Conservation laws}

The dynamics within the system are constrained by the conservation of mass and momentum, and by the supply of free energy that is associated with the exchange fluxes at the system boundary (which is further discussed in Sects. 3.2 and 3.3). In the context discussed here, the mass balances for water, $m_{\mathrm{W}}$, continental mass $m_{\mathrm{s}}$ are determined from the respective mass fluxes of water and sediments:

$\mathrm{d} m_{\mathrm{w}} / \mathrm{d} t=J_{\mathrm{w}, \text { in }}-J_{\mathrm{w}, \text { out }}$
$\mathrm{d} m_{\mathrm{s}} / \mathrm{d} t=J_{\mathrm{s}, \text { in }}-J_{\mathrm{s}, \text { out }}$

where $J_{\mathrm{w}, \text { in }}$ is the generation rate of runoff from effective precipitation (i.e. rainfall minus evaporation, as the latter plays only an indirect role in fluvial erosion and runoff concentration), $J_{\mathrm{w}, \text { out }}$ is the discharge of water from the basin, $J_{\mathrm{s} \text {,in }}$ is 


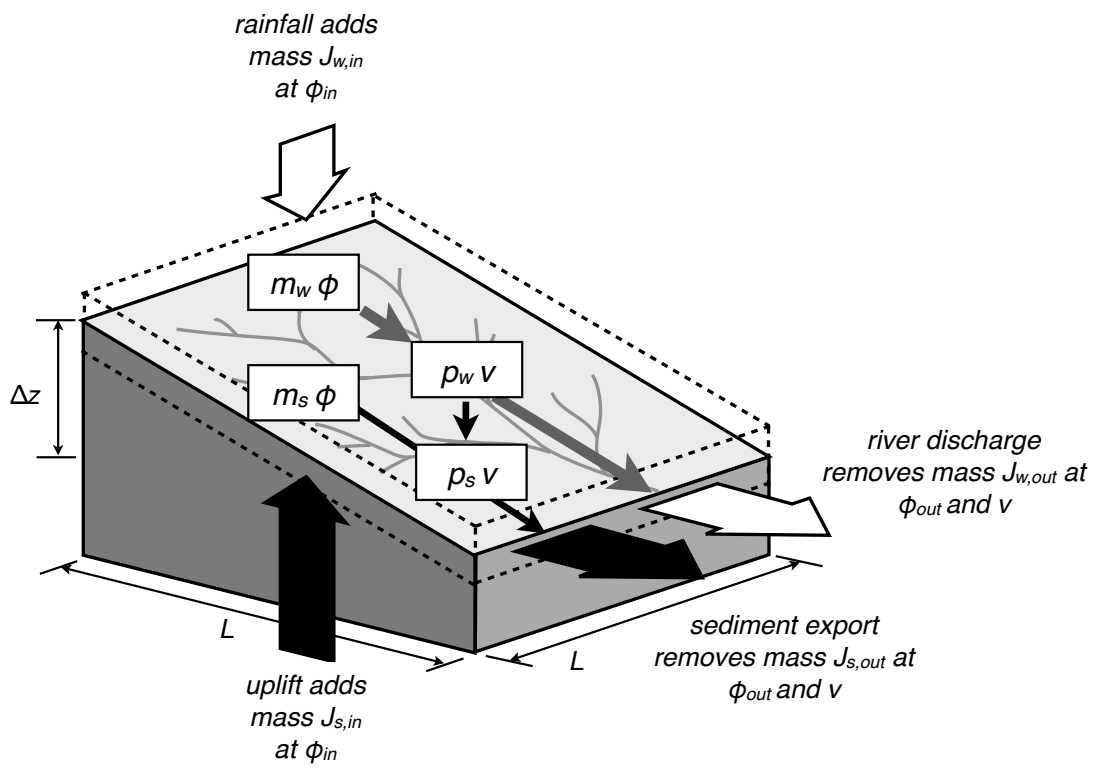

Fig. 3. Definition of a drainage system as a thermodynamic system by delineating its boundaries (dashed lines), the fluxes across the boundaries (in terms of mass and momentum fluxes as well as their respective, conjugate variables), and the four forms of free energy considered in the simple models (potential energies $m_{\mathrm{W}} \phi, m_{\mathrm{s}} \phi$; kinetic energies $p_{\mathrm{W}} v, p_{\mathrm{s}} v$ ). The change in energy within the system is expressed through the respective values of the conjugate variables that convert mass and momentum fluxes to energy exchange fluxes. Ultimately, these energy fluxes set the limits to the strength of the dynamics within the system.

the rate of continental uplift, and $J_{\mathrm{s}, \text { out }}$ is the rate of sediment export (i.e. the net outflux of sediments from the system).

The respective momentum balances for river and sediment flows $p_{\mathrm{w}}$ and $p_{\mathrm{s}}$ are governed by the balance of forces:

$\mathrm{d} p_{\mathrm{w}} / \mathrm{d} t=F_{\mathrm{w}, \text { acc }}-F_{\mathrm{w}, \mathrm{d}}-J_{\mathrm{w}, \text { out }}^{\mathrm{p}}$

$\mathrm{d} p_{\mathrm{s}} / \mathrm{d} t=F_{\mathrm{s}, \text { acc }}+\left(F_{\mathrm{w}, \mathrm{d}}-F_{\mathrm{w}, \text { crit }}\right)-F_{\mathrm{s}, \mathrm{d}}-J_{\mathrm{s}, \text { out }}^{\mathrm{p}}$,

where $F_{\mathrm{w}, \text { acc }}$ and $F_{\mathrm{s} \text {,acc }}$ are the accelerating forces due to geopotential gradients (which for sediments plays a role only for soil creep and detachment in steep terrain), $F_{\mathrm{w}, \mathrm{d}}$ and $F_{\mathrm{s}, \mathrm{d}}$ are the drag forces that act on water and sediment flow, respectively (where $F_{\mathrm{w}, \mathrm{d}}$ includes the drag $F_{\mathrm{w}, \mathrm{s}}=F_{\mathrm{w}, \mathrm{d}}-F_{\mathrm{w}, \text { crit }}$ on sediment that results in its detachment when the drag exceeds a threshold of $F_{\mathrm{w}, \text { crit }}$, and $J_{\mathrm{w}, \text { out }}^{\mathrm{p}}$ and $J_{\mathrm{s}, \text { out }}^{\mathrm{p}}$ are the exports of momentum associated with water and sediment flow. For simplicity we neglect the momentum transferred on sediments by rain splash. We include the threshold term in the momentum balance because this is often found in parameterizations of sediment transport. It plays an important role in determining the magnitude of sediment transport, but it actually is not a critical parameter to understand the conditions under which we would expect the formation of structures, as we will see further below.

The steady state of the mass and momentum balances are given when runoff generation balances river discharge, $J_{\mathrm{w}, \text { in }}=J_{\mathrm{w}, \text { out }}$, continental uplift balances sediment export, $J_{\mathrm{s}, \text { in }}=J_{\mathrm{s}, \text { out }}$, acceleration of water flow balances the drag force and momentum export, $F_{\mathrm{w}, \text { acc }}=F_{\mathrm{w}, \mathrm{d}}+J_{\mathrm{w}, \text { out }}^{\mathrm{p}}$, and the forces acting on the sediment balances the friction force experienced by the sediment and the export of momentum, $F_{\mathrm{s}, \text { acc }}+F_{\mathrm{W}, \mathrm{s}}=F_{\mathrm{s}, \mathrm{d}}+J_{\mathrm{s}, \text { out }}^{\mathrm{p}}$. In the remainder of the manuscript, we consider the steady states of the mass and momentum balances and neglect $F_{\mathrm{s}, \text { acc }}$. The assumption of the steady state is a simplification that may only be valid when a system is considered over sufficiently long periods. It allows us to treat these conservation laws and explore the role of structure formation in a simpler way than if the changes in time are considered as well. Real landscapes may maintain non-steady states in which the time derivatives are comparable to the fluxes. With respect to the steady state assumption of the sediment fluxes, this assumption is very common in geomorphology (Ahnert, 1970; Paik, 2011).

\subsection{Exchange fluxes across the system boundary}

The following exchange fluxes across the system boundary affect the mass and momentum balances and the amount of the total energy within the system (Fig. 3):

- effective precipitation, which adds mass to the system at a rate $J_{\mathrm{w}, \text { in }}$ and at a certain geopotential $\phi_{\mathrm{w}, \text { in }}$. We exclude the fraction of precipitation that is evaporated here and only consider the fraction of precipitation that results in runoff. Hence, the combination of a mass flux at a given geopotential adds potential energy $\mathrm{d}\left(m_{\mathrm{w}} \phi_{\mathrm{w}, \text { in }}\right)$ to the system;

- river discharge, which removes mass from the system at a rate $J_{\mathrm{w}, \text { out }}$ at a certain geopotential $\phi_{\mathrm{w}, \text { out }}$ and with a 
certain momentum $p_{\mathrm{w}}$. This flux removes geopotential energy $\mathrm{d}\left(m_{\mathrm{w}} \phi_{\mathrm{w}, \text { out }}\right)$ and kinetic energy $\mathrm{d}\left(p_{\mathrm{w}} v_{\mathrm{w}}\right)$ from the system;

- continental uplift, which adds continental mass to the system at a rate $J_{\mathrm{s}, \text { in }}$ at a certain geopotential $\phi_{\mathrm{s}, \mathrm{in}}$. This addition of mass at a given geopotential adds potential energy $\mathrm{d}\left(m_{\mathrm{s}} \phi_{\mathrm{s}, \mathrm{in}}\right)$ to the system;

- sediment export associated with river discharge, which removes mass from the system at a rate $J_{\mathrm{s}, \text { out }}$ at a certain geopotential $\phi_{\mathrm{s}, \text { out }}\left(=\phi_{\mathrm{w}, \text { out }}\right)$ and with a certain momentum $p_{\mathrm{s}}$. Sediment export hence exports potential $\mathrm{d}\left(m_{\mathrm{s}} \phi_{\mathrm{s}, \text { out }}\right)$ and kinetic energy $\mathrm{d}\left(p_{\mathrm{s}} v_{\mathrm{s}}\right)$ from the system.

For simplicity, we assume $\phi_{\text {in }}=\phi_{\mathrm{w} \text {,in }}=\phi_{\mathrm{s} \text {, in }}$ (we consider the input of water and sediment at the surface at the same elevation) and $\phi_{\mathrm{out}}=\phi_{\mathrm{w}, \mathrm{out}}=\phi_{\mathrm{s}, \text { out }}$ in the following.

Since the heat balance does not play a central role for the dynamics of drainage systems, we do not consider the whole set of heat fluxes that shape the balances for temperature and entropy, $\mathrm{d}(T S)$, within the system. However, we will keep track of the dissipation within the system. Furthermore, we neglect the import of momentum associated with the uplift of continental crust.

\subsection{Dynamics within the system and its relation to energy conversions}

The hydrologic and geomorphic processes within the system relate to the conversions of potential energy that is added to the system by $J_{\mathrm{w}, \text { in }}$ and $J_{\mathrm{s} \text {,in }}$ to kinetic energy which is exported from the system by $J_{\mathrm{w}, \text { out }}$ and $J_{\mathrm{s} \text {,out }}$ with a lower potential energy. Additionally, some of the kinetic energy is converted to heat. In a simplified treatment we need to account for at least the following processes:

- generation of motion associated with water flow, resulting from an accelerating force $F_{\mathrm{w}, \text { acc }}$, at the expense of depleting its potential energy. That is, the potential energy $\mathrm{d}\left(m_{\mathrm{w}} \phi_{\text {in }}\right)$ is converted into kinetic energy of the form $\mathrm{d}\left(p_{\mathrm{w}} v_{\mathrm{w}}\right)$. When we consider the classical definition of mechanical work as $\mathrm{d} W=F \mathrm{~d} x$, with $\mathrm{d} W=\mathrm{d}\left(m_{\mathrm{w}} \phi_{\mathrm{in}}\right)$, this yields the well-known expression for gravitational acceleration along the slope with an angle $\alpha$ of $F_{\mathrm{w}, \text { acc }}=\nabla\left(m_{\mathrm{w}} \phi_{\text {in }}\right)=m_{\mathrm{w}} g \sin \alpha \approx$ $m_{\mathrm{w}} g \Delta z / L$;

- frictional dissipation of water flow $D_{\mathrm{w}}$, associated with a drag force $F_{\mathrm{w}, \mathrm{d}}$, which is driven by the velocity gradient $\nabla v$ between the water flow and the resting, continental crust. In other words, some of the kinetic energy $\mathrm{d}\left(p_{\mathrm{w}} v_{\mathrm{w}}\right)$ is converted into heat $\mathrm{d}(T S)$;

- the drag force $F_{\mathrm{w}, \mathrm{s}}$ due to the difference in velocities of the water flow and the sediment performs work on the sediment. This work entails, e.g. overcoming of binding forces of the sediment, the lifting of sediment into the water flow, the acceleration to the speed of the flow and its maintenance in suspension against gravity. That is, some of the kinetic energy of the water flow $\mathrm{d}\left(p_{\mathrm{w}} v\right)$ is converted to kinetic energy of the sediment $\mathrm{d}\left(p_{\mathrm{s}} v\right)$, and, to some extent, potential energy and the reduction of (negative) binding energy (the latter two contributions are neglected here). The partitioning of $F_{\mathrm{w}, \mathrm{s}}$ on the different forms of work performed on the sediments depends on material properties of the sediments, slope and on the utilization of available transport capacity. In the following, we assume that a constant threshold stress $F_{\mathrm{w}, \text { crit }}$ is needed to detach sediment, while the remainder maintains the kinetic energy of the moving sediment. Hence, if $F_{\mathrm{w}, \mathrm{s}}$ is smaller than the threshold, no sediment is detached and can be moved;

- frictional dissipation of sediment flow $D_{\mathrm{s}}$. Similar to frictional dissipation of water flow, some of the kinetic energy associated with sediment transport is converted into heat.

These conversions are characterized by the budget equations of the potential and kinetic energies of water and sediments of the basin, $\left(m_{\mathrm{w}} \phi_{\mathrm{w}}\right),\left(m_{\mathrm{s}} \phi_{\mathrm{s}}\right),\left(p_{\mathrm{w}} v_{\mathrm{w}}\right)$ and $\left(p_{\mathrm{s}} v_{\mathrm{s}}\right)$, respectively. At a minimum, they consist of the following terms:

$$
\begin{aligned}
& \mathrm{d}\left(m_{\mathrm{w}} \phi_{\mathrm{w}}\right) / \mathrm{d} t=J_{\mathrm{w}, \text { in }}^{\mathrm{pe}}-P_{\mathrm{w}}-J_{\mathrm{w}, \text { out }}^{\mathrm{pe}} \\
& \mathrm{d}\left(m_{\mathrm{s}} \phi_{\mathrm{s}}\right) / \mathrm{d} t=J_{\mathrm{s}, \text { in }}^{\mathrm{pe}}-P_{\mathrm{s}}-J_{\mathrm{s}, \text { out }}^{\mathrm{pe}} \\
& \mathrm{d}\left(p_{\mathrm{w}} v_{\mathrm{w}}\right) / \mathrm{d} t=P_{\mathrm{w}}-D_{\mathrm{w}}-P_{\mathrm{w}, \mathrm{s}}-J_{\mathrm{w}, \text { out }}^{\mathrm{ke}} \\
& \mathrm{d}\left(p_{\mathrm{s}} v_{\mathrm{s}}\right) / \mathrm{d} t=P_{\mathrm{s}}+P_{\mathrm{w}, \mathrm{s}}-D_{\mathrm{s}}-J_{\mathrm{s}, \text { out }}^{\mathrm{ke}} .
\end{aligned}
$$

In these equations, $J_{\mathrm{w}, \text { in }}^{\mathrm{pe}}$ describes the import rate of potential energy of water associated with the influx of mass $J_{\mathrm{w} \text {,in }}$ at a geopotential $\phi_{\mathrm{in}}, P_{\mathrm{w}}$ describes the conversion of this potential energy into the kinetic energy of water flow, and $J_{\mathrm{w}, \text { out }}^{\mathrm{pe}}$ describes the export of potential energy due to lateral exchange at a geopotential $\phi_{\text {out }}$. Equivalently, $J_{\mathrm{s}, \text { in }}^{\mathrm{pe}}$ describes the import rate of potential energy by the addition of mass $J_{\mathrm{s}, \text { in }}$ at a geopotential $\phi_{\text {in }}$ associated with continental crust through uplift, which is converted into kinetic energy $P_{\mathrm{s}}$ for sediment transport and is depleted by the export of sediments $J_{\mathrm{s}, \text { out }}^{\mathrm{pe}}$ triggered by the water flow at a potential $\phi_{\text {out }}$ (i.e. it is related to the kinetic energy export $J_{\mathrm{s}, \text { out }}^{\mathrm{ke}}$ associated with sediment export). The kinetic energy of water flow is driven by the input of kinetic energy $P_{\mathrm{w}}$, and is depleted by frictional dissipation $D_{\mathrm{w}}$ (related to the friction force $F_{\mathrm{w}, \mathrm{d}}$ and the velocity gradient), the transfer of free energy to sediment transport $P_{\mathrm{w}, \mathrm{s}}$ (related to the drag force $F_{\mathrm{w}, \mathrm{s}}$ and the velocity gradient), and kinetic energy export $J_{\mathrm{w}, \text { out }}^{\mathrm{ke}}$ by river discharge. The kinetic energy associated with sediment transport results from the balance of the free energy input $P_{\mathrm{w}, \mathrm{s}}$, free energy input from the conversion of potential energy of the sediment to kinetic energy $P_{\mathrm{S}}$ (which generally plays a minor role, as described above), frictional dissipation $D_{\mathrm{s}}$ (related to the drag 
force $F_{\mathrm{s}, \mathrm{d}}$ and the velocity gradient between the moving and resting sediment), and the export of kinetic energy by flux $J_{\mathrm{s}, \text { out }}^{\mathrm{ke}}$.

These equations express the conservation of mass, momentum, and energy at a general level for water and sediment flow within a river catchment and act as constraints to the dynamics. At this general level, we can already identify energetic limits to the dynamics that are not apparent from the mass and momentum balances. The transfer of kinetic energy from water to sediment flow is driven by a velocity gradient, but at the same time acts to deplete this gradient. Transferring more and more kinetic energy to sediment transport would at first increase the rate of sediment transport, but eventually, the decrease in kinetic energy of the water flow would slow down the overall export of water and sediment from the drainage basin. Once sediment is transported, it can be arranged into channel networks that have a lower wetted perimeter for a given water flow in relation to a uniform surface, thereby reducing frictional dissipation. It is in the context of such simple considerations that we explore three ways of maximizing the power of sediment transport and its relation to preferential flow structures in the following.

\section{Maximum power in drainage systems and sediment transport}

We consider three models in the following that deal with the transfer of free energy from water flow to sediment transport (model 1), the effect of rearranging sediments into the form of river channels on the overall power to drive the depletion of the topographic gradient (model 2), and the effect of enhanced removal of continental crust by sediment transport on continental uplift (model 3). The three models consider the mass, momentum, and energy balances in steady state, i.e. the fluxes are constant and the state variables do not change in time. Furthermore, we assume that $v_{\mathrm{w}}=v_{\mathrm{S}}=v$ for simplicity. This implies that we neglect bedload transport and focus on the transport of suspended sediments.

\subsection{Model 1: maximum power to drive sediment export}

In the first model we consider the generation and dissipation of kinetic energy associated with surface runoff, and how much work can be extracted from this flow to drive sediment export from the slope. To do so, we consider the mass balances of water and sediments as well as the momentum balance for water flow in a steady state. Since we assume $v_{\mathrm{W}}=v_{\mathrm{s}}=v$, we need to consider only one momentum balance, so our starting point are the three balance equations for $m_{\mathrm{w}}, p_{\mathrm{w}}$, and $m_{\mathrm{s}}$.

We start with the mass balance for $m_{\mathrm{w}}$, which balances effective precipitation with the discharge from the slope:

$\mathrm{d} m_{\mathrm{w}} / \mathrm{d} t=0=J_{\mathrm{w}, \text { in }}-m_{\mathrm{w}} v / L$.
Here, the runoff is expressed as the mass of water on the slope, $m_{\mathrm{w}}=\rho_{\mathrm{w}} L W H$, with $L$ and $W$ being the length and width of the slope and $H$ corresponding to the height of water. Hence, the formulation of runoff is equal to $\rho_{\mathrm{w}} A v$, with $A=W H$ being the cross section of the flow. The mass balance yields an expression for the total mass of water, $m_{\mathrm{w}}$, on the slope as a function of effective precipitation, $J_{\mathrm{w}, \text { in }}$, and the flow velocity of runoff, $v$ :

$m_{\mathrm{w}}=J_{\mathrm{w}, \text { in }} L / v$.

The momentum balance (Eq. 9) for water yields the flow velocity $v$ on the slope:

$\mathrm{d}\left(m_{\mathrm{w}} v\right) / \mathrm{d} t=0=F_{\mathrm{w}, \text { acc }}-F_{\mathrm{w}, \mathrm{d}}-J_{\mathrm{w}, \text { out }}^{\mathrm{p}}$,

where $F_{\mathrm{w}, \mathrm{d}}$ is a drag force on water flow which includes friction and the stress that the water flow applies to the sediment, $F_{\mathrm{w}, \mathrm{s}}$. The accelerating force for water flow on the slope per unit slope length, $F_{\mathrm{w}, \text { acc }}$, depends on the slope (that is, the geopotential gradient $\Delta \phi / L)$ and on the mass of water on the slope (we neglect the effect of the water column on the overall geopotential gradient):

$F_{\mathrm{w}, \mathrm{acc}}=m_{\mathrm{w}} g \sin \alpha \approx m_{\mathrm{w}} \Delta \phi / L=J_{\mathrm{w}, \text { in }} \Delta \phi / v$,

where the approximation is made that for small angles $\sin \alpha \approx \alpha \approx \Delta z / L$. The export of momentum from the slope, $J_{\mathrm{w}, \text { out }}^{\mathrm{p}}$, is given by the mass export (which equals the import in steady state, $J_{\mathrm{w}, \text { out }}=J_{\mathrm{w}, \text { in }}$ ) at a velocity $v$ :

$J_{\mathrm{w}, \text { out }}^{\mathrm{p}}=\left(m_{\mathrm{w}} v\right) v / L=J_{\mathrm{w}, \text { in }} v$.

Without specifying the specific form of the drag force, we can combine Eqs. (17)-(19) and obtain a quadratic equation for $v$ as a function of $F_{\mathrm{w}, \mathrm{d}}$ :

$v^{2}+F_{\mathrm{w}, \mathrm{d}} v / J_{\mathrm{w}, \text { in }}-\Delta \phi=0$

which yields a solution (with the restriction that $v \geq 0$ ) of

$v=\left(F_{\mathrm{w}, \mathrm{d}}^{2} /\left(4 J_{\mathrm{w}, \text { in }}^{2}\right)+\Delta \phi\right)^{1 / 2}-F_{\mathrm{w}, \mathrm{d}} /\left(2 J_{\mathrm{w}, \text { in }}\right)$.

Two limits of this expression can be derived, depending on the relative magnitude of $F_{\mathrm{w}, \mathrm{d}}^{2} /\left(4 J_{\mathrm{w}, \mathrm{in}}^{2}\right)$ and $\Delta \phi$ in the root of Eq. (21). We use the ratio of these two quantities to define a dimensionless number $N_{\mathrm{d}}$ :

$N_{\mathrm{d}}=F_{\mathrm{w}, \mathrm{d}} /\left(2 J_{\mathrm{w}, \text { in }} \Delta \phi^{1 / 2}\right)$.

This dimensionless number expresses the strength of the drag force in relation to the slope, with a large value of $N_{\mathrm{d}}$ representing strong drag on shallow slopes, while a small value of $N_{\mathrm{d}}$ represents little drag on steep slopes. Then, the root in Eq. (21) is expressed as $\Delta \phi^{1 / 2}\left(1+N_{\mathrm{d}}^{2}\right)^{1 / 2}$ and can be approximated for the limit of small $\left(N_{\mathrm{d}} \approx 0\right)$ and large $\left(N_{\mathrm{d}} \gg 1\right)$ values. At the limit of little frictional drag 
$\left(F_{\mathrm{w}, \mathrm{d}} \approx 0\right.$ and $\left.N_{\mathrm{d}} \approx 0\right)$, the root can be approximated by $\Delta \phi^{1 / 2}\left(1+N_{\mathrm{d}}^{2}\right)^{1 / 2} \approx \Delta \phi^{1 / 2}\left(1+N_{\mathrm{d}}^{2} / 2\right) \approx \Delta \phi^{1 / 2}$. This approximation yields the limit for the steady state flow velocity of

$v \approx \Delta \phi^{1 / 2}$.

At the other limit of strong drag, $F_{\mathrm{w}, \mathrm{d}} \gg 2 J_{\mathrm{w}, \text { in }} \Delta \phi^{1 / 2}$ and $N_{\mathrm{d}} \gg 1$, the root in Eq. (21) can be approximated by $\Delta \phi^{1 / 2}\left(1+N_{\mathrm{d}}^{2}\right)^{1 / 2} \approx \Delta \phi^{1 / 2}\left(N_{\mathrm{d}}+1 /\left(2 N_{\mathrm{d}}\right)\right)=F_{\mathrm{w}, \mathrm{d}} /\left(2 J_{\mathrm{w}, \text { in }}\right)$ $+J_{\mathrm{w}, \text { in }} \Delta \phi / F_{\mathrm{w}, \mathrm{d}}$ for large $N_{\mathrm{d}}$. Then, the velocity is approximately

$v \approx\left(J_{\mathrm{w}, \text { in }} / F_{\mathrm{w}, \mathrm{d}}\right) \Delta \phi$.

In this case the drag force strongly interacts with the flow velocity and the dependence of the resulting flow velocity on the slope changes from being proportional to $\Delta \phi^{1 / 2}$ to $\Delta \phi$. Note that Eq. (23) represents supercritical flow, while Eq. (24) can yield expressions for Chezy or Darcy flow. The latter depends on the choice of $F_{\mathrm{w}, \mathrm{d}}$. If $F_{\mathrm{w}, \mathrm{d}}$ is a turbulent, frictional force that depends on the flow velocity, this equation would yield the expression for Chezy flow (in which case the flow velocity would also be proportional to $\Delta \phi^{1 / 2}$ ). If $F_{\mathrm{w}, \mathrm{d}}$ is a binding force that does not depend on the flow velocity, this equation yields an expression for Darcy flow.

Before we explicitly consider the mass balance of suspended sediments, we note that the drag on water flow is needed to provide the stress to detach sediment and bring it into suspension. We express detachment as a threshold process as

$F_{\mathrm{w}, \mathrm{s}}=F_{\mathrm{w}, \mathrm{d}}-F_{\mathrm{w}, \text { crit }}$,

where $F_{\mathrm{w}, \text { crit }}$ is a material-specific threshold stress and $F_{\mathrm{w}, \mathrm{s}}$ is the force involved in detaching sediment. We assume in the following that the critical threshold stress $F_{\mathrm{w}, \text { crit }}$ describes the frictional dissipation of the kinetic energy of water flow that does not relate to the work of sediment detachment, so that we do not account for the frictional drag of water flow additionally. The work performed by this force will then yield the power to detach sediment, $P_{\mathrm{w}, \mathrm{s}}$, which is given by

$P_{\mathrm{w}, \mathrm{s}}=F_{\mathrm{w}, \mathrm{s}} v$.

As it requires work to detach sediment, the rate of sediment detachment should be directly proportional to this power (Bagnold, 1966). The sediment export rate is then obtained from the mass balance of suspended sediments, which involves the detachment work as well as a sedimentation and export rate:

$\mathrm{d} m_{\mathrm{s}} / \mathrm{d} t=0=\mu P_{\mathrm{w}, \mathrm{s}}-m_{\mathrm{s}} / \tau_{\mathrm{s}}-m_{\mathrm{s}} v / L$,

where $\mu$ is a material specific parameter which yields the mass flux of detached sediment for a given power, $\tau_{\mathrm{s}}$ is a time scale at which sediment remains in suspension, and the sediment export flux is written as $m_{\mathrm{s}} v / L$. This mass balance yields a steady state expression for $m_{\mathrm{S}}$ of

$m_{\mathrm{s}}=\mu P_{\mathrm{w}, \mathrm{s}}\left(\tau_{\mathrm{s}} L\right) /\left(L+\tau_{\mathrm{s}} v\right)$

and a sediment export rate $J_{\mathrm{s} \text {,out }}$ of

$$
\begin{aligned}
J_{\mathrm{s}, \text { out }} & =m_{\mathrm{s}} v / L=\mu P_{\mathrm{w}, \mathrm{s}} v /\left(L / \tau_{\mathrm{s}}+v\right) \\
& =\mu\left(F_{\mathrm{w}, \mathrm{d}}-F_{\mathrm{w}, \text { crit }}\right) v^{2} /\left(L / \tau_{\mathrm{s}}+v\right) .
\end{aligned}
$$

In this expression, both, $P_{\mathrm{w}, \mathrm{s}}$ and $v$, depend on the drag force, $F_{\mathrm{w}, \mathrm{d}}$, but in opposing ways. While $P_{\mathrm{w}, \mathrm{s}}$ increases with $F_{\mathrm{w}, \mathrm{d}}$, the terms including $v$ decrease with $F_{\mathrm{w}, \mathrm{d}}$. This results in a maximum possible sediment flux associated with an intermediate value of $F_{\mathrm{w}, \mathrm{d}}$. Figure 4 shows qualitatively the variation of the different terms as well as the maximum in $J_{\mathrm{s} \text {,out }}$ as a function of $N_{\mathrm{d}}$. For the plot, values of $L=100 \mathrm{~m}, J_{\mathrm{w}, \text { in }}=0.01 \mathrm{~kg} \mathrm{~m}^{-2} \mathrm{~s}^{-1}, \Delta \phi=1 \mathrm{~m}^{2} \mathrm{~s}^{-2}, \tau_{\mathrm{s}}=10 \mathrm{~s}$, $\mu=1 \mathrm{~kg} \mathrm{~m}^{-2} \mathrm{~W}^{-1}$, and $F_{\mathrm{w}, \text { crit }}=0.1 \mathrm{~N}$ were used.

We can characterize this maximum in terms of two contrasting limitations, the extent to which sediment is detached, and the ability of the water flow to export the sediment. These two limits are characterized by the ratio of a velocity that is described by the length and time scale of suspended sediments within the system, $v_{\mathrm{s}}=L / \tau_{\mathrm{s}}$, in relation to the velocity of water flow, $v$, and can be expressed by another dimensionless number $N_{\mathrm{s}}$, defined by

$N_{\mathrm{s}}=v_{\mathrm{s}} / v$.

The first limit of low sediment deposition $\left(N_{\mathrm{s}} \approx 0\right.$, or $\tau_{\mathrm{s}} v \gg L$, which means that the effective transport distance before settling is much larger than the basin length) represents the case where the power to detach sediment is limiting the sediment export flux. At this limit, we obtain the approximation

$J_{\mathrm{s}, \mathrm{out}} \approx \mu P_{\mathrm{w}, \mathrm{s}}$

which represents the limit of low values of $N_{\mathrm{d}}$ in Fig. $4 \mathrm{a}$, because a low drag results in high export ability (as reflected by the high value of $v$ ) while detachment of sediments is limited. The other limit is obtained for large values of $N_{\mathrm{s}}$. In this case, $v^{2} /\left(v_{\mathrm{s}}+v\right)=v\left(v / v_{\mathrm{s}}\right) /\left(1+v / v_{\mathrm{s}}\right) \approx v^{2} / v_{\mathrm{s}}$, and

$J_{\mathrm{s}, \mathrm{out}} \approx \mu P_{\mathrm{w}, \mathrm{s}} v / v_{\mathrm{s}}$.

This limit is shown for high values of $N_{\mathrm{d}}$ in Fig. 4a, where due to the high drag, the low flow velocity limits the export of sediments from the system.

We now trace the power that is provided by the generation of potential energy by effective precipitation to drive sediment export from the slope. To start, the power generated by effective precipitation over a geopotential difference $\Delta \phi$ is given by

$P_{\mathrm{w}}=F_{\mathrm{w}, \mathrm{acc}} v=J_{\mathrm{w}, \text { in }} \Delta \phi$, 


\section{a. Sediment Export}

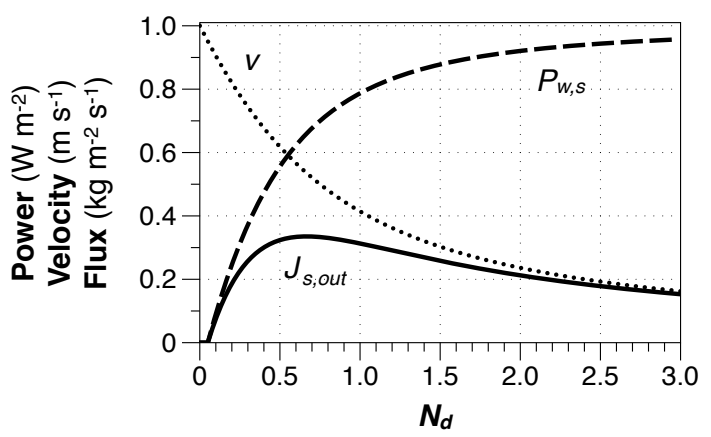

b. Energetics

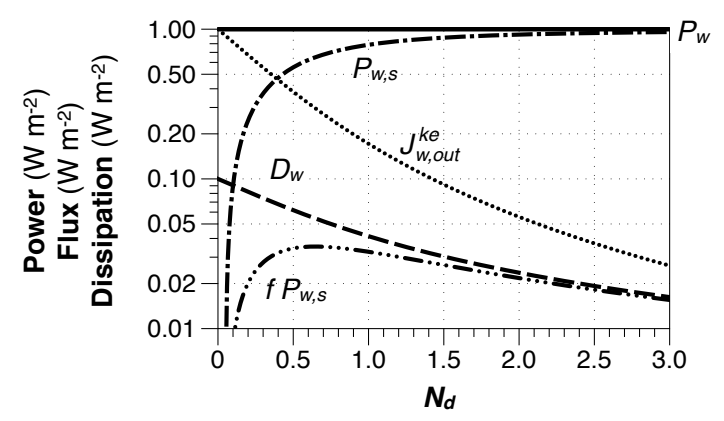

Fig. 4. Demonstration of a maximum rate of sediment export resulting from the tradeoff of increased drag resulting in greater work in detaching sediments, $P_{\mathrm{w}, \mathrm{s}}$, but lower flow velocity, $v$. (a) Water flow velocity $v$, free energy transfer, $P_{\mathrm{w}, \mathrm{s}}$, and rate of sediment export, $J_{\mathrm{S} \text {,out }}$, as a function of the dimensionless number, $N_{\mathrm{d}}$, that characterizes the strength of the drag force, $F_{\mathrm{w}, \mathrm{d}}$, in relation to the accelerating force, $F_{\mathrm{w}, \text { acc }}$, associated with the slope. (b) Sensitivity of total power, $P_{\mathrm{W}}$, frictional dissipation, $D_{\mathrm{w}}$, in water flow, kinetic energy export, $J_{\mathrm{w}, \mathrm{kut}}^{\mathrm{ke}}$, of water flow, and the free energy transfer, $P_{\mathrm{w}, \mathrm{s}}$, from water flow to sediment transport, and the fraction, $f P_{\mathrm{w}, \mathrm{s}}$, that results in sediment export.

which is the well-known expression for stream power when considered in a channel (Bagnold, 1966). A part of this power is wasted by frictional loss, $D_{\mathrm{w}}$, or exported as kinetic energy by runoff, $J_{\mathrm{w}, \text { out }}^{\mathrm{ke}}$, while another part is used to power the detachment of sediment, $P_{\mathrm{w}, \mathrm{s}}$ :

$D_{\mathrm{w}}=F_{\mathrm{w}, \text { crit }} v$

$J_{\mathrm{w}, \text { out }}^{\mathrm{ke}}=J_{\mathrm{w}, \text { out }}^{\mathrm{p}} v=J_{\mathrm{w}, \text { in }} v^{2}$

$P_{\mathrm{w}, \mathrm{s}}=\left(F_{\mathrm{w}, \mathrm{d}}-F_{\mathrm{w}, \text { crit }}\right) v$.

Of the power available for sediment detachment $P_{\mathrm{w}, \mathrm{s}}$, a fraction $f=v /\left(v_{\mathrm{s}}+v\right)=1 /\left(1+N_{\mathrm{s}}\right)$ results in the actual export of sediment by the flux $J_{\mathrm{s}, \text { out }}$, and the remainder $(1-f)$ represents the part of the power that is lost when detached sediments are deposited and return to the bed. The different energetic terms are shown in Fig. 4b, with the fraction of power provided by runoff generation that ends up in sediment export from the slope shown in the graph as $f J_{\mathrm{s} \text {,out }}$.
Table 4. Different dependencies of sediment export, $J_{\mathrm{s}, \text { out }}$, on geopotential difference, $\Delta \phi$, (or slope) for different cases of dimensionless numbers $N_{\mathrm{d}}$ and $N_{\mathrm{s}}$.

\begin{tabular}{llll}
\hline case & $\begin{array}{l}\text { drag on } \\
\text { water } \\
\text { flow } \\
\left(N_{\mathrm{d}}\right)\end{array}$ & $\begin{array}{l}\text { sediment } \\
\text { export } \\
\text { limitation } \\
\left(N_{\mathrm{S}}\right)\end{array}$ & $\begin{array}{l}\text { sediment } \\
\text { export } \\
\left(J_{\mathrm{s}, \text { out }}\right)\end{array}$ \\
\hline A & low & low & $\propto(\Delta \phi)^{1 / 2}$ \\
B & low & high & $\propto(\Delta \phi)$ \\
C & high & low & $\propto(\Delta \phi)$ \\
D & high & high & $\propto(\Delta \phi)^{2}$ \\
\hline
\end{tabular}

The importance of these two limits, as formulated by the two dimensionless numbers $N_{\mathrm{d}}$ and $N_{\mathrm{s}}$, is that the limits yield contrasting dependencies of the sediment export rate $J_{\mathrm{s}, \text { out }}$ on the driving gradient, $\Delta \phi$ (Table 4). In case A of small values of $N_{\mathrm{d}}$ and $N_{\mathrm{s}}$, which describes conditions of low frictional drag and if sediment is being detached, it is easily exported. The expression for the sediment export is obtained by combining Eqs. (25) and (33)

$J_{\mathrm{s}, \mathrm{out}} \approx \mu F_{\mathrm{W}, \mathrm{s}} \Delta \phi^{1 / 2}$.

In this case, the rate of sediment export depends on the root of the slope, $J_{\mathrm{s}, \text { out }} \propto(\Delta \phi)^{1 / 2}$. This case could, for instance, represent the case of the transport of very fine sediment in a channel. In case B of a high value of $N_{\mathrm{d}}$, but a low value of $N_{\mathrm{s}}$, the expression for the sediment export is obtained by combining Eqs. (26) and (33)

$J_{\mathrm{s}, \text { out }} \approx \mu\left(F_{\mathrm{w}, \mathrm{s}} / F_{\mathrm{w}, \mathrm{d}}\right) J_{\mathrm{w}, \text { in }} \Delta \phi$.

Here, $J_{\mathrm{s}, \text { out }} \propto \Delta \phi$. Case $\mathrm{C}$ is represented by a low value of $N_{\mathrm{d}}$, but a high value of $N_{\mathrm{s}}$. The expression for the sediment export is obtained by combining Eqs. (25) and (34)

$J_{\mathrm{s}, \mathrm{out}} \approx \mu\left(F_{\mathrm{w}, \mathrm{s}} / v_{\mathrm{s}}\right) \Delta \phi$.

This case also yields a linear relationship of sediment export, $J_{\mathrm{s}, \text { out }} \propto(\Delta \phi)$, and could represent transport of coarser sediments in a channel. The last case $\mathrm{D}$ is obtained by high values of $N_{\mathrm{d}}$ and $N_{\mathrm{s}}$. By combining Eqs. (26) and (34) we obtain

$J_{\mathrm{s}, \text { out }} \approx \mu\left(F_{\mathrm{w}, \mathrm{s}} / F_{\mathrm{w}, \mathrm{d}}\right)\left(J_{\mathrm{w}, \text { in }} / v_{\mathrm{s}}\right)(\Delta \phi)^{2}$.

This case of strong friction and limited ability to export sediments yields $J_{\mathrm{s} \text {,out }} \propto \Delta \phi^{2}$. This case is representative of overland (or subsurface) flow on relatively shallow slopes. As we will see in the following, this is the most relevant case for structure formation because the non-uniformity in the slope enhances the sediment export rate of the slope.

In summary, model 1 demonstrates that only a small fraction of the power generated by runoff can be utilized to detach and export sediments and thereby deplete the geopotential driving gradient of the slope. The existence of a 
maximum in the sediment export rate results from the fundamental trade-off of increased drag yielding greater sediment detachment, but also inevitably reducing the flow velocity at which sediment is exported. In the case of such strong interactions between water flow and sediment transport, the functional dependence of the sediment export rate on the slope is altered to quadratic form. Even though a maximum rate of sediment export may not be achieved, it is this case of strong interaction and non-linear dependence on slope which will be of most relevance for the discussion of structure formation in Sect. 5 below.

\subsection{Model 2: maximization of sediment export by minimization of frictional losses}

Once work is performed on the sediment, mass can be rearranged to form structures, such as channel networks. The presence of channels will affect the intensity of frictional drag in model 1, as water flow in a channel has less friction per unit volume of runoff compared to overland flow because water in the channel has, on average, less contact to the solid surface at rest. In other words, the formation of a channel will result in shifting the limit of high drag in the case of overland flow towards less drag and hence towards the case of channel flow. This effectively leads to a lower value of $N_{\mathrm{d}}$, and thereby alters the relationship between sediment export and the gradient in geopotential.

The model presented here is set up to show that this difference of flow resistance can minimize frictional dissipation of water flow in the presence of channels, so that sediment can be exported at a higher rate and the export limitation associated with overland flow can be reduced. To do so, we consider a slope of dimension $L$ (length and width) that is wetted uniformly with an effective precipitation $J_{\mathrm{w}, \text { in }}$ and on which the runoff is discharged from the slope through channels. We assume a constant flow velocity $v$ of water and a given drag force $F_{\mathrm{w}, \mathrm{d}}$.

We start by writing the frictional dissipation rate of the water flow $D_{\mathrm{w}}$ as the sum of dissipation by overland flow, $D_{\mathrm{w}, \mathrm{o}}$, and channel flow, $D_{\mathrm{w}, \mathrm{c}}$, respectively:

$D_{\mathrm{w}}=D_{\mathrm{w}, \mathrm{o}}+D_{\mathrm{w}, \mathrm{c}}$.

The frictional dissipation of overland flow, $D_{\mathrm{w}, \mathrm{o}}$, takes place across a contact area of $d_{\mathrm{c}} L$, so that $D_{\mathrm{w}, \mathrm{o}}$ can be expressed as

$D_{\mathrm{w}, \mathrm{o}} \approx D_{\mathrm{w}, 0} d_{\mathrm{c}} L$

where $D_{\mathrm{w}, 0}$ is the constant rate of kinetic energy dissipation of the water flow per unit wetted surface area, $d_{\mathrm{c}}$ is the mean distance to the channel, which is $d_{\mathrm{c}} \approx L /(4 N)$ with $N$ being the number of channels on the slope and $d_{c}=0$ as $N$ approaches infinity $(N \rightarrow \infty)$. This expression is a simplification, as it is only an approximation of the actual flow paths of water to the channel. Note that if we considered subsurface flow in porous media, the contact area would be substantially greater. The actual state of minimum dissipation will be affected by such greater contact area, but the existence of a minimum dissipation state should not be affected.

The dissipation by channel flow, $D_{\mathrm{w}, \mathrm{c}}$, is approximately given by the wetted contact area of the perimeter of the channel, $\pi r_{\mathrm{c}}$, over the length of the slope $L$ :

$D_{\mathrm{w}, \mathrm{c}} \approx D_{\mathrm{w}, 0} \pi r_{\mathrm{c}} N L$,

where $r_{\mathrm{c}}$ is the hydraulic radius of the channel, which is assumed to be a semicircle for simplicity. This radius $r_{\mathrm{c}}$ is determined from the constraint that in steady state, the total flux of water $J_{\text {w,in }}$ is drained through the $N$ channels at bank-full flow:

$J_{\mathrm{w}, \text { in }}=N \rho_{\mathrm{w}} v \pi r_{\mathrm{c}}^{2} / 2$

or

$r_{\mathrm{c}}=\left[2 J_{\mathrm{w}, \mathrm{in}} /\left(\rho_{\mathrm{w}} v \pi N\right)\right]^{1 / 2}$.

Using Eq. (41) to express $r_{\mathrm{c}}$ in $D_{\mathrm{w}, \mathrm{c}}$, we get for the total dissipation rate $D_{\mathrm{w}}$ :

$$
\begin{aligned}
& D_{\mathrm{w}}=D_{\mathrm{w}, 0} L^{2} /(4 N)+D_{\mathrm{w}, 0}\left[2 \pi N J_{\mathrm{w}, \mathrm{in}} /\left(\rho_{\mathrm{w}} v\right)\right]^{1 / 2} L \\
& =a N^{-1}+b N^{1 / 2} .
\end{aligned}
$$

This expression of total frictional dissipation exhibits a minimum value for a certain optimum number of channels, $N_{\text {opt }}$, due to the tradeoff of a decrease in $D_{\mathrm{w}, \mathrm{o}}$ as $N^{-1}$ with a higher number of channels because the distance, $d_{\mathrm{c}}$, to the next channel decreases with $N$, and an increase in $D_{\mathrm{w}, \mathrm{c}}$ with $N^{1 / 2}$ because the total wetted perimeter of all channels increases with increasing $N$. This minimum in frictional dissipation, $D_{\mathrm{w}, \min }$, is found with an optimum number of channels, $N_{\mathrm{opt}}$, to be

$D_{\mathrm{w}, \min }=(3 / 2) \pi^{1 / 3} D_{\mathrm{w}, 0} L^{4 / 3}\left(J_{\mathrm{w}, \mathrm{in}} /\left(\rho_{\mathrm{w}} v\right)\right)^{1 / 3}$

and

$N_{\text {opt }}=(2 a / b)^{2 / 3}=L^{2 / 3}(8 \pi)^{-1 / 3}\left(\rho_{\mathrm{w}} v / J_{\mathrm{w}, \text { in }}\right)^{1 / 3}$.

When we express the water inflow as $J_{\mathrm{w}, \text { in }}=\rho i L^{2}$, where $i$ is the effective precipitation intensity, then it follows that the optimal channel density $N_{\mathrm{opt}}^{3}=v /(8 \pi i)$ depends on the velocity and thus on the slope and the rainfall intensity, $i$. If the stream velocity does not vary too much, then regions with a high rainfall intensity should have a low optimum channel density, while regions with low rainfall intensity should have a high optimum channel density (although channel density is also affected by other factors such as stage of development).

Figure 5 shows qualitatively the variation of the dissipation terms as a function of channel number $N$ and illustrates the minimum dissipation state. For the plot, values of $L=1 \mathrm{~m}, J_{\mathrm{w}, \text { in }}=0.01 \mathrm{~kg} \mathrm{~m}^{-2} \mathrm{~s}^{-1}, \rho_{\mathrm{w}}=1000 \mathrm{~kg} \mathrm{~m}^{-3}$, 
$v=1 \mathrm{~m} \mathrm{~s}^{-1}$, and $D_{\mathrm{w}, 0}=1 \mathrm{~W} \mathrm{~m}^{-2}$ were used. According to this example, an optimum is achieved for $N=16$ (Fig. 5).

In the absence of channels, the frictional dissipation would be $D_{\mathrm{w}}(N=0)=D_{\mathrm{w}, 0} L^{2}$ or $1 \mathrm{~W}$ using the values given in the example. The total frictional dissipation of the whole slope is reduced in the best, optimal case to $22 \%$ (cf. Fig. 5). This minimization in overall frictional dissipation rate is caused by the existence of the channels, so that the work on the channel surface is reduced due to the reduction in drag as compared to the slope, but the transport of sediment is maintained more easily. This reduction of sediment work within the channel enhances the persistence of the structure. It also relates closely to the notion of "minimum energy expenditure" of the optimal river network theory, because the frictional dissipation of kinetic energy of the fluid flow is minimized. Overall, the effect of channel flow is to transport more sediment for the same mean slope $\Delta \phi / L$. Stated differently, the sediment export limitation is reduced, resulting in a lower value of $N_{\mathrm{d}}$ and $N_{\mathrm{s}}$ for the flow within the channel.

\subsection{Model 3: large-scale maximization of topographic gradient depletion}

As sediment is exported by channel flow from land to the sea, the geopotential gradient that drives the flow is slowly depleted, at small scales, but also at the continental scale, reducing the mass of continental crust, $m_{\mathrm{s}}$. As the weight of the continental crust decreases, it experiences isostatic rebound, resulting in continental uplift. In a steady state in which the mass of continental crust does not change, i.e. $\mathrm{d} m_{\mathrm{s}} / \mathrm{d} t \approx 0$, the removal of continental crust by sediment export $J_{\mathrm{s} \text {,out }}$ is balanced by continental uplift, $J_{\mathrm{s} \text {, in }}=J_{\mathrm{s} \text {, out }}$. Formally, we would also need to require that some tectonic process adds continental crust to maintain such a steady state. This steadystate assumption is very common in landscape evolution models (Ahnert, 1970; Paik, 2011). Hence, a higher rate of sediment export in steady state is matched by a greater uplift rate of continental mass. At the same time, however, stronger sediment export results in a diminished geopotential gradient, and a reduced gradient allows for less work to be performed on sediment export. These two contrasting effects, greater uplift with greater sediment export, but greater depletion of the geopotential driving gradient with greater sediment export, result in a trade-off that affects the power associated with the uplift of continental crust. This trade-off shapes the value of the gradient in geopotential $\Delta \phi / L$ that drives runoff and sediment transport.

The third model aims to demonstrate that this trade-off results in a state of maximum power associated with the lifting of continental mass (after Dyke et al., 2011). To start, we consider the mass balance of sediments $m_{\mathrm{s}}$ in steady state (Eq. 8):

$\mathrm{d} m_{\mathrm{s}} / \mathrm{d} t=0=J_{\mathrm{s}, \text { in }}-J_{\mathrm{s}, \text { out }}$,

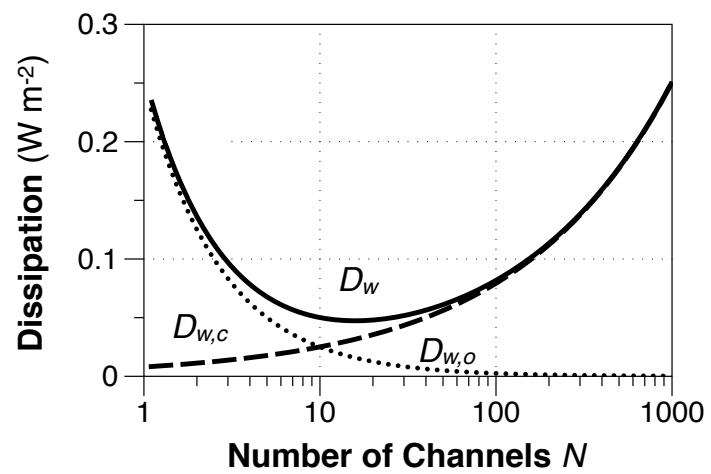

Fig. 5. Demonstration of a state of minimum dissipation of kinetic energy of water flow due to the presence of channels. The graph shows the sensitivity of total dissipation, $D_{\mathrm{w}}$, as well as the two components (dissipation by overland and channel flow, $D_{\mathrm{w}, \mathrm{o}}$ and $D_{\mathrm{w}, \mathrm{c}}$, respectively) to the density of channels, $N$.

where $J_{\mathrm{s}, \text { in }}$ is the rate of uplift, and $J_{\mathrm{s} \text {,out }}$ is the sediment export. We express the rate of uplift, $J_{\mathrm{s}, \text { in }}$, as a form of buoyancy to capture the effect of isostatic rebound as

$J_{\mathrm{s}, \text { in }}=J_{0}-k_{\mathrm{up}} \Delta \phi / L$,

where $J_{0}$ is the rate of uplift without any continental mass above mean sea level (i.e. $\Delta \phi / L=\left(\phi_{\text {in }}-\phi_{\text {out }}\right) / L=0$, so that $\left.J_{\mathrm{s}, \text { in }}=J_{0}\right)$, and $k_{\text {up }}$ is a coefficient that includes the difference in densities of continental crust and the upper mantle. This formulation of the uplift rate follows directly from the associated buoyancy force, which is given by the decrease in the total potential energy with respect to the vertical displacement (see also Appendix). When used in the mass balance for $m_{s}$, one gets an asymptotic relaxation towards isostatic equilibrium when noting that $\Delta \phi \propto m_{\mathrm{s}}$. This expression yields a state of isostatic equilibrium with no uplift when the difference in geopotential is $\Delta \phi_{0} / L=J_{0} / k_{\text {up }}$.

With sediment export, a geopotential gradient $\Delta \phi / L<$ $\Delta \phi_{0} / L$ is maintained away from isostatic equilibrium. This state is associated with continental uplift due to isostatic rebound, and is associated with the generation of potential energy, $J_{\text {s,in }}^{\text {pe }}$ (or, alternatively, the power involved in continental uplift) given by

$J_{\mathrm{s}, \text { in }}^{\mathrm{pe}}=J_{\mathrm{s}, \text { in }} \Delta \phi / L$.

Using the steady state $\left(J_{\mathrm{s}, \text { in }}=J_{\mathrm{s} \text {,out }}\right)$ and Eq. (46), we can write $\Delta \phi / L$ as

$\Delta \phi / L=\left(J_{0}-J_{\text {s,out }}\right) / k_{\text {up }}$

so that the generation of potential energy associated with the lifting of continental crust becomes

$J_{\mathrm{s}, \text { in }}^{\mathrm{pe}}=J_{\mathrm{s}, \text { out }}\left(J_{0}-J_{\mathrm{s}, \text { out }}\right) / k_{\mathrm{up}}$.

This expression has a maximum value of

$J_{\mathrm{s}, \text { in,max }}^{\text {pe }}=J_{0}^{2} /\left(4 k_{\mathrm{up}}\right)$ 
for a sediment export rate of $J_{\mathrm{s} \text {,out }}=J_{0} / 2$ and an associated, optimum geopotential difference of $\Delta \phi_{\mathrm{opt}} / L=\Delta \phi_{0} /(2 L)$. This trade-off between the uplift rate and the height at which the continental mass is lifted to is shown in Fig. 6 as well as the resulting state of maximum power. For the plot, values of $k_{\mathrm{up}}=1 \mathrm{~kg} \mathrm{~s} \mathrm{~m}^{-3}$ and $U_{0}=1 \mathrm{~kg} \mathrm{~m}^{-2} \mathrm{~s}^{-1}$ were used. The existence of a maximum power state can be understood as follows. With small sediment export, a state near isostatic equilibrium is being maintained, the gradient in geopotential is near its maximum value. In this state, little new potential energy is generated by uplift because the state is near isostasy. With greater values of sediment export, more potential energy is being generated, but the steady state gradient in geopotential is maintained at a lower value. For very large sediment fluxes, the gradient in geopotential is decreased such that the generation of potential energy by uplift is decreased. However, since sediment export is driven by this gradient, it seems implausible that steady states are maintained beyond the maximum power state.

Since the geopotential difference generated by uplift is depleted only by sediment transport in these considerations, the maximum power state of uplift corresponds to the maximum intensity of depleting the geopotential difference in steady state. Maximum power of uplift occurs when the sediment transport rate is proportional to $J_{0} / 2$.

\subsection{Maximum power and interactions between the three models}

To set these three models into the larger context, let us revisit the continental view shown in Figs. 1 and 2 and relate this view to the fundamental question of how the depletion of the geopotential gradients in topography generated by geological processes is accelerated by the free energy input from the water cycle. The thermodynamic formulation of this perspective in Sect. 3 included the balance equations of mass and momentum for water and sediment transport (Eqs. 710 ), and the associated forms of potential and kinetic energy (Eqs. 11-14). The conservation of mass in steady state yields the almost trivial insight that $J_{\mathrm{w}, \text { in }}=J_{\mathrm{w}, \text { out }}$ and $J_{\mathrm{s}, \text { in }}=J_{\mathrm{s} \text {,out }}$. It is not trivial because the steady states can be achieved at different magnitudes of fluxes and by different intensities of interactions. Different magnitudes of the fluxes are in turn associated with different rates of energy conversions and, ultimately, these differ in the rate at which the geopotential driving gradient is being depleted.

With Model 1 we derived different limits on sediment transport from a given rate of effective precipitation $J_{\mathrm{w}, \text { in }}$ and geopotential gradient $\Delta \phi / L$. Two of the limits concerned the strength of frictional drag $F_{\mathrm{w}, \mathrm{d}}$ in relation to the accelerating force $F_{\mathrm{w}, \text { acc }}$ of water flow that is due to the geopotential gradient. These limits resulted in different functional relationships of water flow velocity $v$ to slope and relate to the well-established hydrological transport laws of supercritical flow at the one extreme, and water flow in porous media

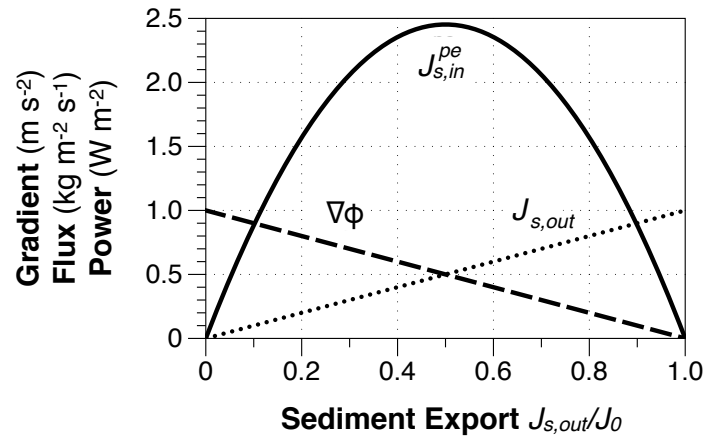

Fig. 6. Demonstration of a state of maximum power associated with continental uplift through sediment export. The graph shows the sensitivity of the power associated with uplift, $J_{\mathrm{s} \text {,in }}^{\mathrm{pe}}$, as well as the decrease in slope, $\nabla \phi$, to the intensity of sediment export, as expressed by the ratio $J_{\mathrm{s} \text {,out }} / J_{0}$.

at the other extreme. When the intensity of drag is then further related to the rate at which work is being performed to detach sediments, two limits were obtained in which either the detachment limits sediment export or the deposition of sediments within the system. Again, these two limits relate to the well-established limits of detachment and transport limits in sediment transport. What we show here is that these two limits are associated with different functional dependencies on velocity, and thereby on slope. At the detachment limit, the rate of sediment export is proportional to the flow velocity while at the other limit at which the rate of deposition of suspended sediment limits export, the rate of sediment export is proportional to the square of the flow velocity. Combined with the two limits on flow velocity, this results in a range of functional dependencies of sediment export on slope ranging from an exponent of $1 / 2$ to 2 . These different dependencies originate from different intensities of interaction between water flow and sediment transport. It is at the limit of high drag and low ability to export sediments when the system has the greatest ability to redistribute sediments within the system (i.e. to build and maintain channel structures) and thereby affecting the relative importance of these limits. Model 1 also demonstrates that a maximum in sediment export exists at intermediate values. This maximum can be understood as a state of co-limitation in which both limitations, detachment and deposition, act in similar strength on sediment export, thereby resulting in the maximum export of sediments.

Once such channel flow structures are shaped, Model 2 showed that the presence of channels can reduce the frictional drag on water flow in relation to the gravitational acceleration. Hence, the formation of channel structures can alter the high drag, low export limit and shift it towards the low drag, high export limit that would be characterized by lower values of $N_{\mathrm{d}}$ and $N_{\mathrm{s}}$. Thereby, the system exports the detached sediments at a faster rate. As the relative contribution to frictional dissipation is a combination of overland 
and channel flow, a minimum in frictional loss can be obtained at a certain channel density $N$. This optimum channel density decreases with increasing mass of water that is to be exported. Hence, as larger and larger continental regions are being considered that drain greater volumes of water and sediments, the formation of greater and fewer drainage channels can reduce the frictional losses further, and thus enhance sediment export and the depletion of continental-scale geopotential gradients.

With increasing values of sediment export, the geopotential gradient is brought further and further away from a state of isostatic equilibrium. That is, the lo$\mathrm{cal}$, isostatic disequilibrium of the geopotential gradient $\Delta \phi_{0} / L-\Delta \phi / L=J_{\mathrm{s}, \text { out }} / k_{\text {up }}$ (cf. Eq. 46) increases with increasing values of $J_{\mathrm{s}, \text { out }}$, and results in greater rates of continental uplift. This reduction in gradient, however, inevitably reduces the power $P_{\mathrm{w}}$ and $P_{\mathrm{S}}$ that drive sediment export. Model 3 showed that through adjustments in the intensity of sediment export, continental uplift can be maintained in a state of maximum power at which the generation rate of potential energy of continental crust at the surface is at a maximum. Through this effect, the driving gradient for sediment transport, $\Delta \phi / L$, is maintained at a higher value in steady state than in the absence of isostatic rebound.

In summary, the three models taken together sketch out how the input of free energy by the continental water cycle can accelerate the dynamics that deplete the state of isostatic equilibrium of the continental crust (Fig. 1b) towards a state of global equilibrium (Fig. 1d). This acceleration of continental sediment export is not arbitrary, but strictly bound by upper limits on how much free energy can be transferred from runoff to sediment transport and from isostatic rebound. Furthermore, the reduction in frictional dissipation associated with channel flow provides a means to understand how the overall system could achieve such an optimum state at which these upper limits are reached. This leaves the question as to why the dynamics should progress towards these upper limits, which we will address in the following section.

\section{Evolution towards disequilibrium and maximization by structure formation}

The three models of the previous section establish the limits to the dynamics of sediment transport, the importance of interactions, and the ingredients to understand how maximization associated with the depletion of geopotential gradients could be achieved. We now make the link between the three models more explicit. We discuss how the evolution of a drainage system from a uniform slope to a structured basin, as shown in Fig. 7, can be understood as the expected and inevitable outcome of the dynamics that evolve to maximize the dissipation of the driving geopotential gradient by the export of sediment from the system.
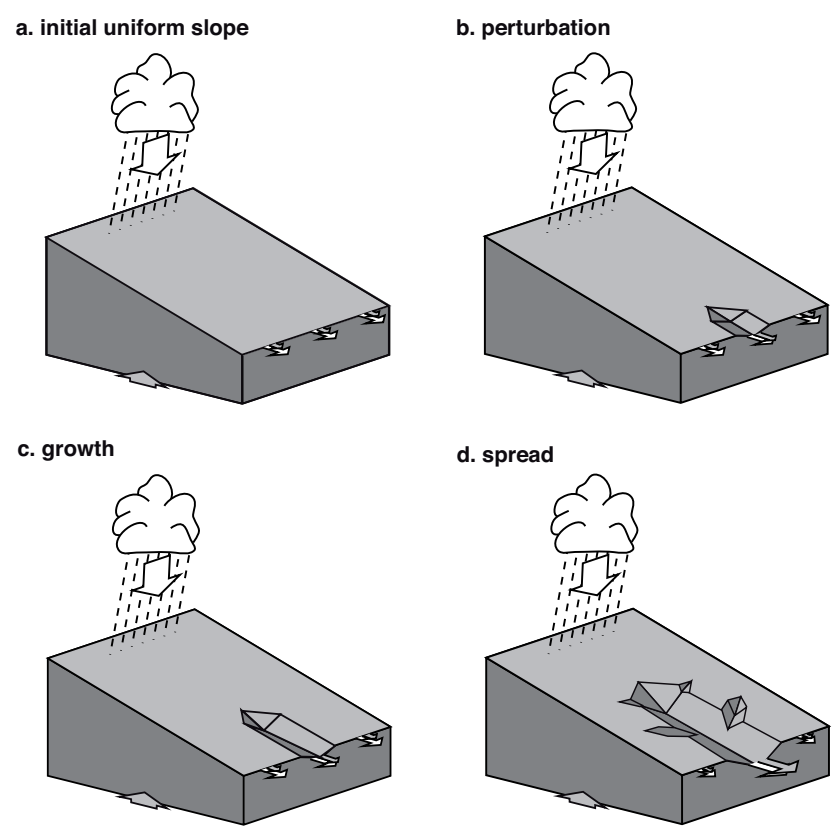

d. spread
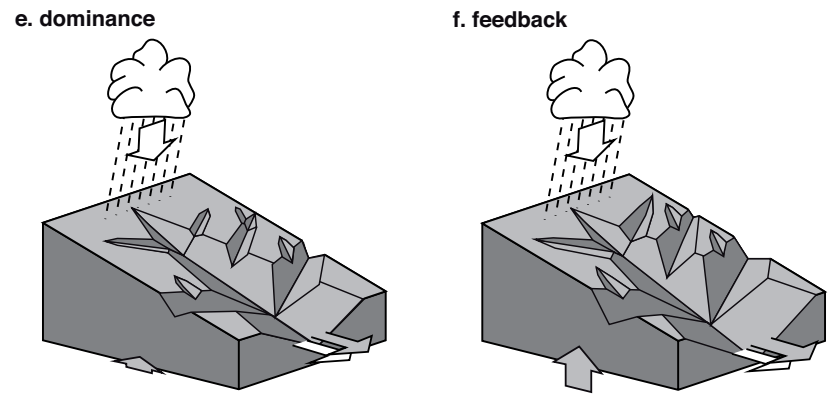

Fig. 7. Six stages of structure formation that reflect increasing levels of disequilibrium and ability to generate free energy and drive sediment transport. See main text for description.

In this example, our use of the term "structure" refers to the spatial, topographic heterogeneity on the slope and includes the combination of two aspects: (a) the nonuniformity (or heterogeneity) of the geopotential gradients, expressed by the deviation of the local slopes from the mean slope, and (b) the arrangement of these local deviations occurs in an ordered way by the ordered, backward-invasive process of channel incision. Hence, our use of "structure" not only includes the connected channel network, but also the steepened slopes that frame the channel network. In other words, we refer to "structure" as organized heterogeneity along connective pathways.

To do so, we consider a thought experiment, in which we look at a uniform and homogeneously sloped surface that is close to isostatic equilibrium and experiences very little uplift (Fig. 7a). This slope is in a steady-state with respect to the mass balances of water and sediments, i.e. the net influx of water and sediments into the system balances the export of runoff and sediments. The sequence of steps of how a 
drainage network may form is shown in Fig. 7b-f. We use this thought experiment to discuss qualitatively the increase in energy conversions as well as the feedbacks that result in structure formation. The evolutionary steps are consistent with the insights that were derived from the models of the previous section. The example is hypothetical, but also resembles channel structures that are commonly found in nature. Note that along real rivers, the slope does not vary uniformly, but is typically concave-upward (Smith and Bretherton, 1972). For simplicity, we use a uniform slope here.

Before we describe these steps in more detail, we note that the evolution in structure shown in Fig. 7 is mostly reflected in the heterogeneity of the geopotential gradient rather than its mean value and by the connectivity of this heterogeneity into the channel network structure. We first introduce a measure of spatial disequilibrium that captures the variation in the driving gradient (that is, the heterogeneity in the slope). This measure describes the spatial extent of the structure. We then relate this measure to the enhanced sediment export, and describe the energetics of structure formation. The relation of this measure of disequilibrium to the connectivity of the structure is discussed at the end of Sect. 5.1 and in more detail in Sect. 5.3.

\subsection{Disequilibrium associated with structure}

The heterogeneity associated with the presence of a structure relates to a non-uniform distribution of the geopotential driving gradient $\nabla \phi_{i}$ across the slope (where we use the $\nabla$ symbol to refer to the local gradient), with the index $i$ used to refer to a particular location on the slope. This gradient plays the central role to drive sediment export, as shown by model 1. Depending on which limit acts on sediment export, the extent of heterogeneity on the slope has different implications on the magnitude of sediment export.

Let us consider a simple example to illustrate the contrasting role of heterogeneity in sediment export. We represent the heterogeneity in gradients by only two values, $\nabla \phi_{1}$ and $\nabla \phi_{2}$, of equal abundance with $\nabla \phi_{1}=\nabla \phi+\nabla \phi_{\mathrm{h}}$ and $\nabla \phi_{2}=\nabla \phi-\nabla \phi_{\mathrm{h}}$, where $\nabla \phi$ is the mean gradient of the slope $(\nabla \phi=\Delta \phi / L)$ and $\nabla \phi_{\mathrm{h}}$ represents the deviation from the mean gradient associated with heterogeneity. In the case of open channel flow (i.e. small values of $N_{\mathrm{d}}$ and $N_{\mathrm{s}}$ ), the rate of sediment export $J_{\mathrm{s}, \text { out }}$ depends on $\nabla \phi^{1 / 2}$. In this case, the sediment export decreases with increasing heterogeneity $\nabla \phi_{\mathrm{h}}$ :

$$
\begin{aligned}
J_{\mathrm{s}, \text { out }} & \propto 1 / 2\left(\nabla \phi_{1}^{1 / 2}+\nabla \phi_{2}^{1 / 2}\right) \\
& \approx \nabla \phi^{1 / 2}\left(1-1 / 4 \nabla \phi_{\mathrm{h}}^{2} / \nabla \phi^{2}\right),
\end{aligned}
$$

where the Taylor approximations $(1+x)^{1 / 2} \approx 1+x / 2-x^{2} / 8$ and $(1-x)^{1 / 2} \approx 1-x / 2-x^{2} / 8$ for small values of $x=\left(\nabla \phi_{\mathrm{h}} / \nabla \phi\right)$ were used. Because $J_{\mathrm{s}, \text { out }}$ decreases with $\nabla \phi_{\mathrm{h}}$, this expression implies that heterogeneity will result in less sediment export, and that a decrease in heterogeneity will result in enhanced sediment export. A maximum in sediment export is reached in the case of $\nabla \phi_{\mathrm{h}}=0$, i.e. a uniform distribution of slope within the channel. Since frictional dissipation by water flow in this limit also depends on $\nabla \phi^{1 / 2}$ (cf. Eq. 34), the maximum in sediment export corresponds to a minimum of frictional dissipation by water flow. This minimum in energy dissipation is consistent with the assumptions made by optimal river networks of minimum energy dissipation or expenditure.

In contrast, in the case of overland flow, i.e. large values of $N_{\mathrm{d}}$ and $N_{\mathrm{s}}$, the rate of sediment export $J_{\mathrm{s} \text {,out }}$ depends on $\nabla \phi^{2}$. In this case we find that the heterogeneity in the slope enhances sediment export:

$J_{\mathrm{s}, \text { out }} \propto 1 / 2\left(\nabla \phi_{1}^{2}+\nabla \phi_{2}^{2}\right)=\nabla \phi^{2}+\nabla \phi_{\mathrm{h}}^{2}$.

In other words, the case where high detachment of sediment and high deposition within the slope represents the situation in which a rearrangement of the driving gradient within the slope enhances the sediment export. As the slope is altered, this affects the local value of $N_{\mathrm{d}}$, with a steepening of the slope resulting in a lower value of $N_{\mathrm{d}}$ for the same intensity of drag. It is this case that is of central relevance for the formation of the structures shown in Fig. 7.

To discuss structure formation, we use the deviation of the local gradient $\nabla \phi_{i}$ from the mean gradient $\nabla \phi$ as a basis to define a measure of spatial disequilibrium $D \phi$ associated with the presence of a structure:

$D \phi=\left(\int\left(\nabla \phi_{i}^{2}-\nabla \phi^{2}\right) \mathrm{d} A / A\right)^{1 / 2}$,

where the integration has taken over the area, $A$, of the whole slope. This measure includes both, areas that exhibit steeper slopes than the mean as well as areas with slopes shallower than the mean. Note that this measure of disequilibrium is insensitive to the spatial arrangement of the deviations. A random arrangement of these deviations could result in the same measure as a spatial arrangement of interconnected channels of a flow network. As the latter configuration exhibits a stronger organization, this disequilibrium measure by itself is insufficient to detect persistent structures. When we look for persistent structures, we essentially look for a disequilibrium $D \phi$ that grows and persists in time. In other words, we look for those spatial arrangements of disequilibrium that are associated with a positive feedback on its own growth. It is only through such a positive feedback that the disequilibrium can develop and persist in time.

\subsection{Dynamics of structure formation}

To describe the dynamics of structure formation, we first conceptually separate the area of the slope $A$ into those parts that reflect the structure (the heterogeneous part of the slope, with 
both, steeper and shallower slopes than the mean), $A_{\text {structure, }}$ and those of the remaining parts of the slope (the uniform part of the slope), $A_{\text {slope }}$ :

$A=A_{\text {structure }}+A_{\text {slope }}$.

The spatial extent of the structure, $A_{\text {structure }}$, represents those areas in which the local slope deviates from the mean slope by a certain threshold value and thus includes the channel network. Then, the sediment export characteristics of the whole slope can be separated into the contribution to the total sediment export by the structure, $J_{\mathrm{s}, \text { out,structure }}$ and by the sediment export of the mean properties of the slope, $J_{\mathrm{s} \text {,out,slope }}$ for the remaining area $A_{\text {slope }}$.

Since the sediment export from the structure, i.e. the combination of steeper hillslopes and less steep channels, is on average greater than the export from the remaining slope, the depletion of potential energy of the sediment should differ. Hence, we separate the depletion of potential energy into two terms, one representing the depletion of the potential energy of the structure, $\left(m_{\mathrm{S}} \phi_{\mathrm{S}}\right)_{\text {structure }}$, and one for the remaining slope, $\left(m_{\mathrm{S}} \phi_{\mathrm{S}}\right)_{\text {slope }}$ :

$\mathrm{d}\left(m_{\mathrm{s}} \phi_{\mathrm{s}}\right) / \mathrm{d} t=\mathrm{d}\left(m_{\mathrm{s}} \phi_{\mathrm{s}}\right)_{\text {structure }} / \mathrm{d} t+\mathrm{d}\left(m_{\mathrm{s}} \phi_{\mathrm{s}}\right)_{\text {slope }} / \mathrm{d} t$.

We can then further express the individual changes of potential energy by

$\mathrm{d}\left(m_{\mathrm{s}} \phi_{\mathrm{s}}\right)_{\text {structure }} / \mathrm{d} t=\left(J_{\mathrm{s}, \text { in }} \phi_{\text {in }}-J_{\mathrm{s}, \text { out structure }} \phi_{\text {out }}\right) A_{\text {structure }} / A$

and

$\mathrm{d}\left(m_{\mathrm{s}} \phi_{\mathrm{s}}\right)_{\text {slope }} / \mathrm{d} t=\left(J_{\mathrm{s}, \text { in }} \phi_{\text {in }}-J_{\mathrm{s}, \text { out }, \text { slope }} \phi_{\text {out }}\right) A_{\text {slope }} / A$,

where we assume that both components are governed by the same rate of uplift, $J_{\mathrm{s}, \text { in }}$, but differ in their rates of sediment export.

Since the initial state of the slope shown in Fig. 7a most likely represents the case of overland flow, the rate of sediment export will be proportional to $\nabla \phi_{i}^{2}$. Because the structure by definition reflects the part of the slope with heterogeneity, it will have a greater rate of sediment export, so that the depletion rate of potential energy of the structure should proceed at a greater rate than that of the remaining slope. That is, $\left|\mathrm{d}\left(m_{\mathrm{s}} \phi_{\mathrm{s}}\right)_{\text {structure }} / \mathrm{d} t\right|>\left|\mathrm{d}\left(m_{\mathrm{s}} \phi_{\mathrm{s}}\right)_{\text {slope }} / \mathrm{d} t\right|$, with the difference between the two rates being roughly proportional to $D \phi^{2}$. On the other hand, the different rates of change in $\left(m_{\mathrm{s}} \phi_{\mathrm{s}}\right)_{\text {structure }}$ and $\left(m_{\mathrm{s}} \phi_{\mathrm{s}}\right)_{\text {slope }}$ affect the topographic gradient and the spatial extent of the structure and the surrounding slope, so that this should result in accompanying changes in the areal extent of the structure, $A_{\text {structure, }}$, and the state of disequilibrium, $D \phi$, of the slope.

\subsection{Evolution towards greater disequilibrium and structure}

The evolutionary trend in Fig. 7 is characterized by the areal extent of the channel network structure, $A_{\text {structure }}$, and its disequilibrium, $D \phi$. The different stages should furthermore reflect clear and consistent trends in the variables that reflect the intensity by which the geopotential gradient is depleted. These include the reduction of frictional dissipation of water flow by overland flow because increasingly more water is exported from the slope through the channel network, which is captured by the two variables $D_{\mathrm{w}, \mathrm{o}}$ and $D_{\mathrm{w}, \mathrm{c}}$. The steepened slopes at the boundary of the structure as well as the reduced frictional dissipation within the channel network of the structure should result in more work done to detach sediments on the steepened slopes and more efficient export of sediments by channel flow from the slope, which is captured by the variables $J_{\mathrm{s} \text {,out,structure }}$ and $J_{\mathrm{s} \text {,out,slope }}$. The trends in these variables is sketched qualitatively in Fig. 8 and described in more detail in the following.

Stage 1: uniformity (Fig. 7a). The initially uniform and homogeneously sloped surface has a uniform gradient in geopotential, so that $\nabla \phi_{i}=\nabla \phi$ at every location $i$, so that $D \phi=0$. Hence, the runoff generated from incoming precipitation experiences the same, high drag throughout the slope which is characterized by a high value of $N_{\mathrm{d}}$. The resulting water flow is dissipated entirely by overland flow as no channels are present, i.e. $D_{\mathrm{w}}=D_{\mathrm{w}, \mathrm{o}}$. As shown in model 2 above, this configuration of flow has the greatest contact with the sediment at the surface and experiences the greatest frictional dissipation. With the greatest intensity of friction, the forces acting on the sediment are greatest as well, but because of the resulting slow flow velocity of overland flow, the actual transport of sediments is small. Hence, little of the continental mass is transported downslope by the flow, and if so, only for a short distance. Overall, this results in little export of kinetic energy of the overland flow as well as little sediment export from the slope $\left(J_{\mathrm{s}, \text { out }} \approx 0\right)$. In steady state, this small flux of sediment export would be balanced by a small rate of continental uplift $\left(J_{\mathrm{s}, \text { in }} \approx 0\right)$, which would involve little power to sustain $\left(J_{\text {s,in }}^{\text {pe }} \approx 0\right)$.

Stage 2: perturbation (Fig. 7b). We now consider a random perturbation that leads to the removal of some sediment from a small area on the slope. This removal has the greatest probability to occur at the lower end of the slope, as this is the place where the highest flux of water per unit cross section occurs. It could, however, also occur further upslope, or at a different location along the slope since we consider a random perturbation. Such a perturbation would lead to a steepening of the local slope, so that $\nabla \phi_{j}>\nabla \phi$ for this location $j$. Our measure for disequilibrium becomes greater than zero, $D \phi>0$ and the area of the structure, while small, starts to be greater than zero, $A_{\text {structure }}>0$. Since the conditions of drag and sediment transport are characterized by high values of $N_{\mathrm{d}}$ and $N_{\mathrm{s}}$, sediment export is proportional to $\left(\nabla \phi_{j}\right)^{2}$. This 
local steepening of the slope hence results in disproportional enhancement of sediment transport from the perturbed area and the local enhancement of sediment export should act to enhance the growth of the perturbation.

Stage 3: growth (Fig. 7c). The enhanced sediment export from the locally steepened slope has two important consequences: first, it forms a positive feedback on the growth of this perturbation. When the locally enhanced export removes material from the steepened slope, it pushes the steepened slope further upslope, where more sediment can be removed. This then acts to enhance the perturbation in spatial extent, resulting in larger values of $A_{\text {structure }}$ and $D \phi$. Second, the area downslope of the steepened slope represents a confined spatial channel structure with a reduced gradient within the channel structure and a reduced contact area to volume flow ratio. That is, drag is reduced, the value of $N_{\mathrm{d}}$ is decreased, while enhancing the ability to export sediment, i.e. the value of $N_{\mathrm{s}}$ is reduced as well. Overall, this results in an enhanced export of kinetic energy of water flow through the channel as well as enhanced export of sediments within the flow. As some water is exported by the structure, the frictional dissipation by overland flow, $D_{\mathrm{w}, \mathrm{o}}$, is reduced, while the sediment export by the structure, $J_{\mathrm{s}, \text { out,structure, }}$ is enhanced.

Stage 4: spread (Fig. 7d). As the steepened slope progresses to grow further upslope and deepens, the slopes along the channel are steepened as well. This steepening of the channel slopes makes them more susceptible to perturbations that remove sediments. The location of such a perturbation would be another random event, with a higher probability to occur in areas of steeper slopes. When such a perturbation arises, this perturbation would grow and experience the same positive feedback as discussed in the previous two steps. This is essentially a self-similar process forming self-similar network structures and it would act to spread the steepening of the gradient in geopotential beyond areas directly upslope of the channel, increasing the values of $A_{\text {structure }}$ and $D \phi$. This growth of the structure would collect more of the generated runoff of the slope, it would generate more work in detaching sediments on the steepened slopes at the edges of the structure, and the channel network within the structure would export water and sediments more effectively. The overall frictional dissipation is decreased, with $D_{\mathrm{w}, \mathrm{o}}$ decreasing substantially, while $D_{\mathrm{w}, \mathrm{c}}$ slightly increasing simply because more water is transported by channels. As more work on detaching sediments is performed and sediments are exported more effectively from the slope, the overall export of sediments, $J_{\mathrm{s}, \text { out }}$, should increase due to the increase in $J_{\mathrm{s} \text {,out,structure }}$.

Stage 5: dominance (Fig. 7e). Eventually, the structure spreads by the positive feedbacks on growth over the whole slope. At this point, $A_{\text {structure }} \approx A, A_{\text {slope }} \approx 0$, and the extent of disequilibrium $D \phi$ has increased further. As the structure grows in size, it becomes more efficient at exporting runoff and sediments, as discussed in the context of model 2 in terms of the sensitivity to $J_{\mathrm{w}, \text { in }}$. This effect results in further reduction in frictional losses within the structure, although the overall dissipation should still somewhat increase due to the increase in size compared to the previous stage. At this stage, the structure composed of steepened gradients at the edges and reduced gradients within the channel network dominates the fluvial behavior of the slope. The steepened slopes at the edges generate more power to provide more work to detach sediments from the slopes. At the same time, the leveling of slope and the reduction in wetted perimeter within the channels enhances the overall export of sediments from the slope. These effects should thus further enhance the overall export of sediments by the structure, $J_{\mathrm{s} \text {,out,structure }}$.

Stage 6: feedback (Fig. 7f). As the structure efficiently erodes and transports the sediment from the slope, its total mass is reduced and so is its weight. With this reduction of mass, the mean slope is being reduced, and thereby the driving force for runoff generation and sediment transport. This reduction in slope thereby acts as a negative feedback to the growth of the structure. On the other hand, the reduction of weight at sufficiently large scales will bring the elevation out of a state of isostatic equilibrium (cf. Fig. 1), which will enhance continental uplift to restore the equilibrium height, as shown by model 3 . While the overall size of the structure can no longer increase as it already dominates the slope, the disequilibrium $D \phi$ can potentially increase further due to the greater uplift of continental crust. Such an increase in $D \phi$ could then affect frictional dissipation as it alters the local gradients, and it can further increase the overall export of sediments from the slope due to the increase in uplift. This, in total, enhances the overall depletion of the topographic gradient between continental and oceanic crust, thereby accelerating the evolution to the global equilibrium state shown in Fig. 1d.

\subsection{Disequilibrium, structures, and maximization}

To sum up, the evolutionary sequence of channel network formation as shown in Figs. 7 and 8 should follow a consistent trend towards greater power for fluvial processes that are able to enhance the sediment export from the region. This trend is accompanied with an evolution towards greater values of spatial disequilibrium, as introduced in Sect. 5.1. Furthermore, the dynamics are such that they inevitably result in greater connectivity of the channel network. At the center of this evolutionary sequence are feedbacks that enhance sediment export by the formation of structure. These feedbacks we explore in the following in more detail.

\section{Time scales and feedbacks}

To better identify the feedbacks that lead to the evolutionary dynamics towards maximization through structure formation, we first introduce two time scales that describe the dynamics described above. We then relate these time scales to the dominant feedbacks that are involved in the maximization 


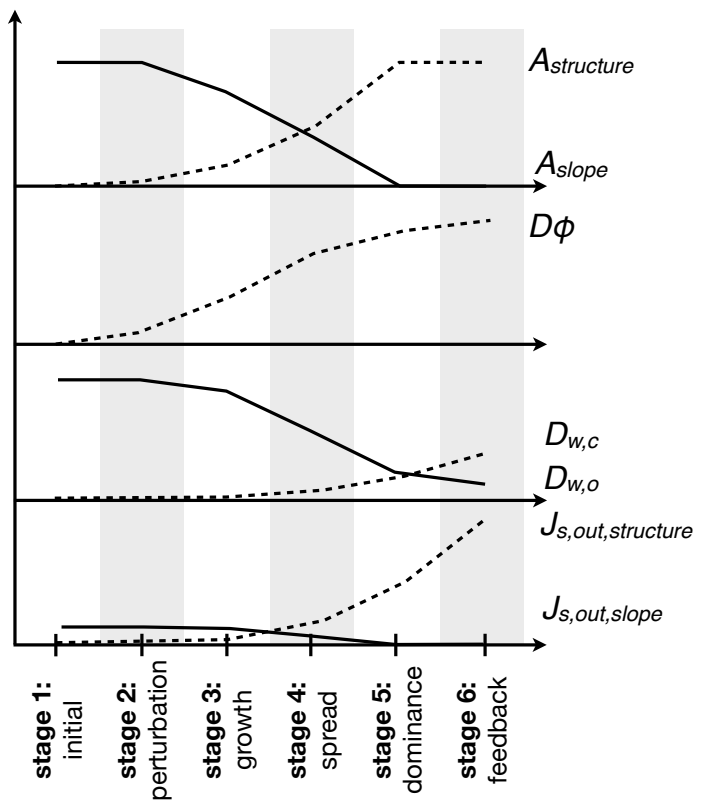

Fig. 8. Qualitative sketch of the change in variables associated with river network structure formation in relation to the different stages shown in Fig. 7. Shown are from top to bottom: the areal extent of the structure, $A_{\text {structure, }}$ in relation to the remaining area of the slope, $A_{\text {slope }}$ the disequilibrium, $D \phi$, of the local geopotential gradient, $\nabla \phi_{i}$, in relation to the mean gradient of the slope, $\nabla \phi$; the frictional dissipation by overland flow, $D_{\mathrm{w}, 0}$, and by channel flow, $D_{\mathrm{w}, \mathrm{c}}$; and the resulting sediment export by the structure,

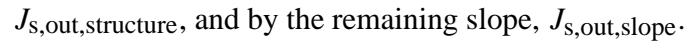

of power to drive the depletion of geopotential gradients at the fastest possible rate.

\subsection{Time scales and structure formation}

The processes involved in structure formation and gradient depletion should be governed by two dominant time scales: a time scale that characterizes the formation of the structure, $\tau_{\text {structure, }}$, and a time scale, $\tau_{\text {depletion }}$, that characterizes the depletion of the geopotential gradient of the slope.

Since potential energy is depleted faster within the structure, the time scale at which structure is formed is described by the build-up of the difference in potential energy between the structure, $\left(m_{\mathrm{s}} \phi_{\mathrm{s}}\right)_{\text {structure, }}$, and the remaining slope, $\left(m_{\mathrm{S}} \phi_{\mathrm{s}}\right)_{\text {slope }}$, in relation to the difference in sediment export from the structure, $J_{\mathrm{s}, \text { out,structure, }}$ to the mean slope, $J_{\mathrm{s}, \text { out,slope }}$ :

$$
\begin{aligned}
\tau_{\text {structure }}= & \left(\left(m_{\mathrm{s}} \phi_{\mathrm{s}}\right)_{\text {slope }}-\left(m_{\mathrm{s}} \phi_{\mathrm{s}}\right)_{\text {structure }}\right) / \\
& \left(J_{\mathrm{s}, \text { out } \text {,structure }} \phi_{\text {out }}-J_{\mathrm{s}, \text { out,slope }} \phi_{\text {out }}\right) .
\end{aligned}
$$

The differences have been arranged such that the sign of $\tau_{\text {structure }}$ is greater than zero. The time scale $\tau_{\text {structure }}$ is not necessarily a fixed value throughout the evolutionary sequence shown in Fig. 7, but may change as the disequilibrium increases. With the progressive development of the disequilibrium, the difference in potential energy increases and so does the value of the nominator, but since the sediment export from the structure increases as well so does the value of the denominator. If the sediment export from the mean slope is small, as is the case when $N_{\mathrm{S}}$ is high, then the respective increases in $\left(m_{\mathrm{s}} \phi_{\mathrm{s}}\right)_{\text {slope }}-\left(m_{\mathrm{s}} \phi_{\mathrm{s}}\right)_{\text {structure }}$ and $J_{\mathrm{s}, \text { out,structure }}$ should proceed at similar paces, so that $\tau_{\text {structure }}$ likely remains relatively constant in time.

The time scale at which the geopotential gradient of the slope is depleted is characterized by the total sediment export from the slope $J_{\mathrm{s} \text {,out }}$ that depletes the overall potential energy of the slope, $\Delta\left(m_{\mathrm{s}} \phi_{\mathrm{s}}\right)_{\mathrm{s}}$, i.e. at geopotential heights above $\phi_{\text {out }}$. The time scale of gradient depletion, $\tau_{\text {depletion, }}$, should hence be expressible as

$\tau_{\text {depletion }}=\Delta\left(m_{\mathrm{s}} \phi_{\mathrm{S}}\right)_{\mathrm{s}} /\left(J_{\mathrm{s}, \text { out }} \phi_{\text {out }}\right)$.

This time scale is not a fixed property either. While the overall potential gradient of the slope changes relatively little while the value of $J_{\mathrm{s} \text {,out }}$ increases through the evolutionary stages of the structure, the time scale should decrease as the formation of the structure progresses.

When we compare the two time scales, we can separate two different cases, $\tau_{\text {structure }}>\tau_{\text {depletion }}$ and $\tau_{\text {structure }}<\tau_{\text {depletion. }}$. The first case represents a case in which no structure can be formed because the driving gradient is depleted faster than the time it would take to form a structure. This case is not of interest here as it does not correspond to a case where a persistent structure has an effect on the depletion of a gradient. We are interested in the other case in which $\tau_{\text {structure }}<\tau_{\text {depletion. }}$. This should be the case when the sediment export is highly limited and $J_{\mathrm{s} \text {,out }} \approx J_{\mathrm{s} \text {,out,structure. }}$ In this case, the denominator has the greatest value in Eq. (58) and since $\left(\left(m_{\mathrm{s}} \phi_{\mathrm{S}}\right)_{\text {slope }}-\left(m_{\mathrm{S}} \phi_{\mathrm{S}}\right)_{\text {structure }}\right)$ should be less than $\Delta\left(m_{\mathrm{S}} \phi_{\mathrm{s}}\right)_{\mathrm{s}}$, the condition $\tau_{\text {structure }}<\tau_{\text {depletion }}$ should be met in this case. It is this case in which structures are formed faster than gradients are depleted. In the following discussion on feedbacks we focus on this latter case.

\subsection{Feedbacks, structure formation, and maximization}

We now discuss how the evolutionary dynamics of drainage basins described in Sect. 5 can be generalized in a scheme of the basic feedbacks involved in the evolutionary dynamics towards a state of maximum power and maximum gradient depletion. This general scheme is shown in Fig. 9 and explained in the following.

In general, a state of maximum power requires at least two feedbacks for the evolutionary dynamics (Ozawa et al., 2003; Kleidon et al., 2012a). A fast, positive feedback starts the dynamics and amplifies these further by generating free energy for the dynamics that deplete the gradient (loop A in Fig. 9). Eventually, a negative feedback results from the generated dynamics from the accelerated depletion of the driving gradient (loop B in Fig. 9). This negative feedback also makes the maximum state stable to perturbations (Ozawa et 


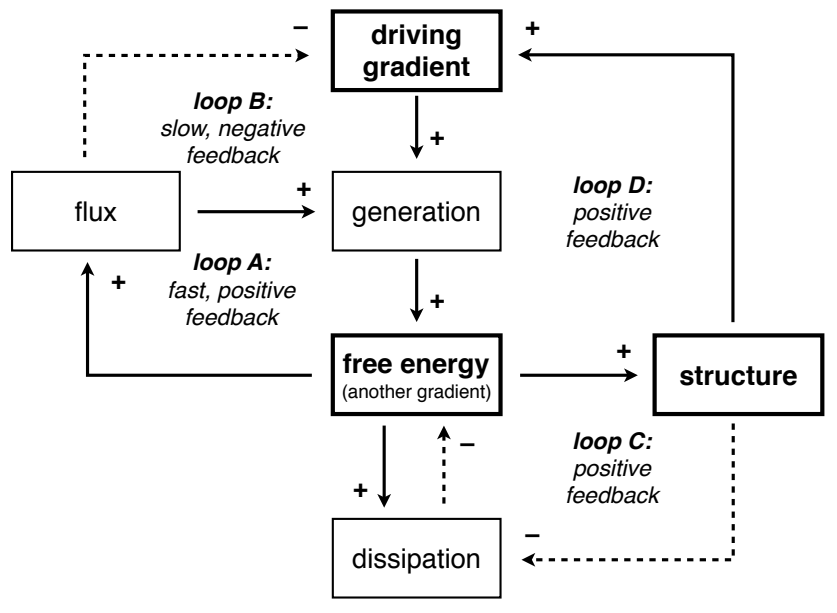

Fig. 9. A general feedback diagram to illustrate how the dynamics of free energy generation enhance gradient depletion and how these dynamics relate to structure formation. Solid lines with "+" indicate positive influences (e.g. a larger driving gradient results in a greater generation rate). Dashed lines with "-" show negative influences (e.g. an enhanced flux reduces the driving gradient). Four feedback loops (A, B, C, D) are shown: Feedbacks A and B on the left relate to the maximum power limit, and the feedbacks $C$ and $D$ on the right relate to how structured flow can achieve this limit. After Kleidon et al. (2012a).

al., 2003). If a small perturbation increases the flux, the driving gradient is reduced, thereby resulting in less generation of free energy and thereby a reduced flux. Likewise, when a small perturbation reduces the flux, the driving gradient is increased and so is the generation rate of free energy. Hence, these two feedbacks would seem to be the minimum ingredients in understanding how systems can evolve to a state of maximum power and why the maximum power state is stable against perturbations.

When applied to the dynamics of sediment transport discussed here, the driving gradient corresponds to the gradient in geopotential of the slope, while the generated free energy relates to the disequilibrium formed in form of a river network structure. The positive feedback that is represented by loop A in Fig. 9 implies that the power for sediment export is enhanced by the resulting dynamics of sediment export. This positive feedback is, in fact, accomplished by the structure formation of two effects: first, the structure is associated with the formation of channeled flow which reduces the dissipative loss (loop C), and second, the steepening of the driving gradient at the edge of the structure locally enhances the driving gradient (loop D). As these two feedbacks act at a time scale $\tau_{\text {structure }}$ of structure formation, these should represent the fast, positive feedback. The negative feedback (loop B) relates to the depletion of the geopotential driving gradient by the enhanced sediment export through structure formation. This feedback acts on the time scale $\tau_{\text {depletion }}$ of gradient depletion.

\section{Discussion}

The models considered here are, of course, extremely simple, with assumptions being made that may not always hold and many details being excluded from the considerations. The steady state assumption that we made for the models may not always hold, in particular because rainfall does not occur uniformly in time but shows distinct temporal variability. Configurations of river networks may not always be in an optimal state, either because they are still evolving and/or because environmental conditions have changed. We also did not consider that continental crust needs to be weathered before it can be transported as sediments, the details of flow paths of water through the soil and the groundwater system before reaching the channel or the uptake of soil water by root systems and subsequent evaporation into the atmosphere. These processes also convert energy. Weathering requires energy to overcome the binding energy of rock minerals, soil water movement and groundwater flow dissipate potential energy, while a root system performs work in lifting water to the canopy. These aspects would need to be considered in a more complete view of energy conversions in catchments, as well as their spatial organization, and their variation across different climates. We also assumed that the sediments are already weathered, so that the limiting process in depleting topographic gradients is to transport the sediments downstream. If this were not the case, and the continental surface would consist mostly of unweathered rock, then the generation of sediments by weathering of primary rock would be the limiting process. Since the biota strongly affects weathering rates (e.g. Dietrich and Perron, 2006, and discussion below), we would then need to include other processes to describe the limitations in depleting gradients in continental topography (and the driving gradients for other processes, such as biotic activity, as well). Additionally, this work must obviously be implemented and utilized for concrete predictions in the future and tested against the rich data that is available associated with the structure of river networks in nature. What we presented here should only be seen as a proof-of-concept and can, therefore, only form the first step.

Nevertheless, the thermodynamic perspective described here - from the basics of energy transfers as the central core of any dynamics of Earth system processes, the three simple models, the qualitative description of river network evolution, and the association of the evolutionary dynamics with two contrasting feedbacks - forms a self-consistent, complete picture which emphasizes the critical importance of a "complete" view of river networks within the Earth system. This complete view requires more than the fundamental conservation of mass and momentum to describe the dynamics of river networks. After all, surface water and sediment at rest conserve mass and momentum just as much as highly dynamic river networks with high rates of sediment transport. The additional constraint on the dynamics arise from the accounting of the associated conversion rates of energy that 
drive the dynamics, and the recognition that these conversion rates are subjected to maximum power limits. This maximum power limit is fundamental. Sediment transport requires free energy for the work needed to detach, lift and transport, but the utilization of the energy source will inevitably impact its strength. The maximum power limit emerges from the transfer of momentum from water flow to sediment work and inevitably must reduce the momentum of the water flow through the conservation of momentum. It is this fundamental trade-off, the increase in power with a greater flux but a decline in power with increased depletion of the driving gradient that results in the maximum power limit. This two-way interaction of a driver causing a flux, but the flux depleting the driver is what we mean by a "complete" view of the dynamics of river networks. What we have shown here with very simple models is that such strong interaction can explain the emergence of structures as a way to deplete gradients at a faster rate. Even though we omitted many aspects in this study, it would seem that the representation of such strong interactions between driver and flux is critical for understanding the presence of structures and for predicting their effects. Hence, it is essential to properly account for the free energy that is generated, transferred and dissipated across processes. When free energy is utilized to drive one flux like sediment detachment - the free energy of another process - river flow - needs to be depleted. It is through this coupling of free energy that the processes that shape river networks interact with other Earth system processes, specifically with the dynamics of the continental crust and water cycling, as shown in Figs. 1 and 2. It is the evaluation of the dynamics that differentiates the one extreme case of surface water and sediment at rest from the other extreme of high sediment transport in terms of their ability to deplete the driving gradient of continental topography. The presence of river network structures can then be seen as a consequence of the second law of thermodynamics in that these structures accelerate the depletion of the driving gradient associated with continental topography.

Our line of reasoning is consistent with previous work. Our very simple treatment of the mass and momentum balances of water in the context of model 1 yielded the well-known transport laws as limits related to the relative strengths of drag in relation to gravitational acceleration. When extended to sediment transport, these yield the two well-established detachment and transport limits of sediment transport (e.g. Whipple and Tucker, 2002). When combined, we were able to show that the rate of sediment export can show contrasting functional relationships on slope, with exponents ranging from $1 / 2$ to 2 . These relationships emerge from different intensities of interactions between water and sediment flow. The different exponents explain the contrasting effects of heterogeneity on the rate of sediment export, where the lack of heterogeneity maximizes sediment export for an exponent of $1 / 2$ with a uniform flow velocity in channel flow with minimum energy dissipation, while the formation of heterogeneity maximizes sediment export for exponents greater than 1 , which is consistent with the steepened hillslopes we find in drainage basins. Note that we do not consider different sediment sizes and increased discharge that would be needed to derive a decrease in slope of river networks and a uniform transport capacity.

The fast, positive feedback that is associated with structure formation (cf. Fig. 9) is consistent with what Phillips (2010) refers to as "hydraulic selection" (although Phillips, 2010, did not associate these feedbacks with optimality, we place such kind of feedbacks into the broader context of thermodynamic directions). Similar feedbacks have also been identified in other systems. For instance, Lenton (1998) identified a positive feedback on growth in terms of coupled populationenvironment dynamics. It would seem that this close association of positive feedbacks and structure formation with enhanced free energy generation is a very general phenomenon and could explain the omnipresence of structures in many environmental systems. These feedbacks do not necessarily need to be formulated in thermodynamic terms to understand their relation to structure formation in environmental system. However, formulating these in thermodynamic terms provides us with the most basic and quantitative basis, so that we can formulate principles of structure formation in the most general terms with the widest range of applicability and relate them to the evolutionary direction set by the second law of thermodynamics. It would thus seem that a thermodynamic systems approach such as the one we have taken here could be used to explore the general role of structure for the dissipative intensity of environmental systems, but this would need to be explored in more detail in the future.

The focus on maximum power that we pursue here contradicts the substantial work related to minimization of energetic attributes, such as stream power (Howard, 1990), dissipation (West et al., 1997) or energy expenditure (RodriguezIturbe and Rinaldo, 1997), only at first sight. Effective precipitation generates the kinetic energy of runoff, which is then either transported downslope, dissipated by friction, or transferred to perform work to detach and transport sediments. Hence, the minimization of frictional dissipation of kinetic energy does not contradict the maximization of sediment export. Likewise, the reduction, or even minimization of frictional dissipation by channel flow (as demonstrated by model 2) is associated with the maximization of transport. While the particular choice of which aspect is minimized or maximized seems arbitrary, it is again the larger scale context which provides the key about the choice of optimization. After all, the processes involved in river network formation are all driven by the geopotential gradients of continental topography, and are directed towards depleting these gradients. It is in this broader context that these processes accelerate the dynamics of geopotential gradient depletion, i.e. they maximize the depletion of thermodynamic gradients to the extent that is possible given the mass and momentum balance constraints. What this emphasizes is that the definition of the 
system boundary and the processes that act within the system is critical to evaluate whether processes within a system minimize or maximize dissipation.

Our work also relates closely to Bejan's suggested "constructal law of nature" (Bejan, 1997). Bejan's suggested law states that "for a finite-size system to persist in time its configuration must change such that it provides easier access to its currents" (Bejan, 1997). Much of the description of Bejan's work relates to the maximization of power, which in part is accomplished by the minimization of frictional loss (e.g. see "engine and brake" discussion in Fig. 2 in Bejan and Lorente, 2011). Our description of structure evolution in Sect. 5 is essentially consistent with Bejan's work, in that the river network evolves in such a way that it enhances overall flux of sediment through the structure. This results in the positive feedback of structure formation as shown in Fig. 9. The description provided here extends Bejan's work in that it (a) provides the basis to actually quantify these trends in terms of fluxes, power and dissipation, and does not need to rely on an ill-defined concept of "access" and (b) that it provides the context of the thermodynamic limits as it relates to the setting of the river network structure within the Earth system.

The description of the evolutionary dynamics of river network of Sect. 5 and the relation to feedbacks that were described in Sect. 6 would need to be evaluated at a quantitative level. It would seem that the outcome should be compatible with previous approaches that evaluate instabilities in the equations of water and sediment transport (e.g. Kirkby, 1971; Smith and Bretherton, 1972; Izumi and Parker, 2000; Smith, 2010), but do not explicitly look at how such instabilities relate to changes in the energetics. It would seem that the fast, positive feedback on structure growth described here would directly correspond to the instabilities found in the previous approaches. In that sense, it seems that our approach is consistent with these previous studies, but this consistency would need a more detailed evaluation in the future.

What we have not considered here is the role of the biota in shaping the dynamics of drainage systems. Dietrich and Perron (2006) have identified biotic contributions to practically all processes that drive the shaping of the continental landscape, such as enhancement of weathering by the biota or slope stabilization by vegetation (see also Phillips, 2009). Yet the models that we developed here are general in establishing the physical limits of the flow of water and sediment and their relation to structure formation within which the biota can have an effect (under the given assumptions of sufficient sediment availability). In this sense, the derivation of the limits, particularly with respect to model 1 in Sect. 4.1, should hold. It would seem instructive to explore biotic effects in a thermodynamic context in future work. We could then ask whether biotic effects would accelerate the dynamics of drainage basins, thereby resulting in a topographic signature of life that is associated with more dissipative drainage basins.

\section{Summary and conclusions}

We described a thermodynamic perspective of the dynamics of river networks in a highly simplistic, but self-consistent view to argue that the evolution and maintenance of river flow structures reflect the fundamental tendency of nature to dissipate gradients as fast as possible. The fastest possible rates for the dynamics are set by the maximum power limit, which was illustrated in the context of three simple models related to drainage systems. The first model described the limits that shape the rate of sediment export and demonstrated a maximum rate of sediment export that would deplete geopotential gradients at the fastest possible rate. The second model showed that channel flow reduces frictional dissipation. The third model showed that on large spatial and temporal scales, the interaction of sediment export with uplift can result in a maximum rate of continental uplift. We then described how the evolution of river network structures can be understood as the implementation of the maximization. Steepened gradients at the edges of the structure disproportionately enhance power generation, while the reduction of frictional dissipation within the structure enhances the export from the structure. We related two basic feedbacks to the evolutionary dynamics of structure formation, with a fast acting, positive feedback by which the growth of the structure enables further growth, and a slow, negative feedback that relates to the depletion of the driving gradient by the dynamics associated with structure formation. This description of structure formation in terms of generation and dissipation of free energy as well as the associated feedbacks is very general and should also be applicable to a broad range of structures that we observe in nature.

In conclusion, our work emphasizes the importance of taking a complete view on Earth systems from a broader, thermodynamic perspective that focuses on energy transformations. The focus on such energy transfers is not an alternative view of how nature works, it needs to be considered at the same fundamental level as the conservation laws of energy, mass, and momentum. The free energy that is generated to drive the dynamics of a particular process needs to come from somewhere and needs to be drawn from these balances. This inevitably not only results in interactions at the small scale but also at the Earth system scale at large, as illustrated in Fig. 2. In the case explored here, it is the drag term in the water flow momentum balance that not only provides the driving force for sediment detachment and export, but also the means to further slow down water flow. It is through the strength of such interactions that the limits on how much free energy can be generated to drive the dynamics of a process are determined and hence these play a central role for the dynamics. Since we can then explain the formation of structures as "enhancers" of the dynamics, it shows how important it is to explore structures and interactions from the perspective of the energetics that are involved in the processes. It should be possible to extend the 
insights gained here to explain structure and heterogeneity in other natural systems, such as preferential flow paths in soils, groundwater or rooting networks, and evaluate the extent to which optimum structures for such processes would differ across different climatic regions.

There are a few practical implications related to these insights for the modeling of drainage systems. First, there may be some deficiencies in model parameterizations regarding the adequate representation of free energy transfer between processes. For instance, the drag applied to water flow not only results in some frictional dissipation by turbulence but also the power to detach sediments. If the latter aspect is ignored in a parameterization of fluid turbulence, then the intensity of turbulence will be overestimated for a given drag. A second implication of this work is that the assumption that processes operate at states of maximum power could potentially be useful in providing a simple and principled way to derive subgrid-scale parameterizations of the effects of heterogeneity for models of land surface hydrology and geomorphology. After all, what we show here is that heterogeneity cannot be ignored and simply averaged out as it can play a critical role in accelerating the dynamics of a process. The extent to which the complexity of channel network formation can be represented by a parameterization derived from maximum power would, however, need to be further explored. Last, the thermodynamic limits derived here could be developed further to derive the maximum rate by which water can drain from a catchment through a river channel network. Furthermore, it should be possible to develop a similar approach to derive the thermodynamic limit of the evaporation flux. The combination of these two approaches would yield the partitioning of precipitation into evaporation and runoff that reflects the fastest depletion of gradients. By comparing this partitioning to the Budyko curve, this may shed some light on the causes for why this partitioning can be described in rather simple terms. This would, however, require further development of this approach.

\section{A1 Reduction in potential energy of continental and oceanic crust from a state of local, isostatic equilibrium to a global, stratigraphic equilibrium state}

In this Appendix we demonstrate that sediment transport from land to ocean results in the reduction of potential energy of continental crust material. To do so, we consider a simple configuration that is shown in Fig. 1. A block of continental crust of length, $L_{\mathrm{c}}$, with density $\rho_{\mathrm{c}}$ rests within oceanic crust of a higher density, $\rho_{\mathrm{o}}$, and length, $L$. The vertical position of the block of continental crust is given by the vertical extent, $\Delta z_{\mathrm{c}}=\Delta z_{\mathrm{c}, 1}$, from a reference line (lower dashed line in Fig. 1a). The thickness of the oceanic crust is considered with regard to its vertical extent, $\Delta z_{1}=\Delta z_{0,1}$, taken from the same reference line. The indices " $\mathrm{c}$ " and "o" refer to continental and oceanic crust, while the indices "l" and "g" refer to the local isostatic equilibrium shown in Fig. 1a and the global, stratigraphic equilibrium shown in Fig. 1c.

To show the reduction in potential energy due to lateral sediment transport, we consider the conservation of mass of the total mass of continental and oceanic crust, which set the constraints on the vertical extents, and the changes in potential energy within the system.

\section{A1.1 Mass balance constraints}

We assume in this example that the mass of both, continental and oceanic crust, $m_{\mathrm{c}}$ and $m_{\mathrm{o}}$, are being conserved.

The mass of continental crust, $m_{\mathrm{c}}$, is given by the density $\rho_{\mathrm{c}}$ as well as the dimensions of the block. In the configuration shown in Fig. 1a, this mass is determined by

$m_{\mathrm{c}}=\rho_{\mathrm{c}} L_{\mathrm{c}} \Delta z_{\mathrm{c}, 1}$,

where for simplicity we assume that the third dimension is included in the density $\rho_{\mathrm{c}}$. For a given mass $m_{\mathrm{c}}$, this translates into an expression for $\Delta z_{\mathrm{c}, 1}$ of

$\Delta z_{\mathrm{c}, 1}=\frac{m_{\mathrm{c}}}{\rho_{\mathrm{c}} L_{\mathrm{c}}}$.

Similarly, the mass of oceanic crust, $m_{\mathrm{o}}$, is given by the density $\rho_{\mathrm{o}}>\rho_{\mathrm{c}}$ and the dimensions:

$m_{\mathrm{o}}=, \rho_{\mathrm{o}}\left(L-L_{\mathrm{c}}\right) \Delta z_{\mathrm{o}, 1}$.

For a given mass of oceanic crust, $m_{\mathrm{o}}$, this yields the vertical extent of the crust, $\Delta z_{\mathrm{o}, 1}$ :

$\Delta z_{\mathrm{o}, 1}=\frac{m_{\mathrm{o}}}{\rho_{\mathrm{o}}\left(L-L_{\mathrm{c}}\right)}$.

When continental crust is redistributed to the state shown in Fig. 1c, the mass of continental crust is given by

$m_{\mathrm{c}}=\rho_{\mathrm{c}} L \Delta z_{\mathrm{c}, \mathrm{g}}$

and the vertical extent changes to $\Delta z_{\mathrm{c}, \mathrm{g}}$ :

$\Delta z_{\mathrm{c}, \mathrm{g}}=\frac{m_{\mathrm{c}}}{\rho_{\mathrm{c}} L}$.

Likewise, the mass of oceanic crust is given by

$m_{\mathrm{o}}=\rho_{\mathrm{o}} L \Delta z_{\mathrm{o}, \mathrm{g}}$

and the vertical extent changes to $\Delta z_{\mathrm{og}, \mathrm{g}}$ :

$\Delta z_{\mathrm{o}, \mathrm{g}}=\frac{m_{\mathrm{o}}}{\rho_{\mathrm{o}} L}$.

A1.2 Potential energy in local, isostatic equilibrium

The potential energy of the configuration shown in Fig. 1a is given by the contributions by continental crust, $U_{\mathrm{pe}, \mathrm{c}, \mathrm{l}}$, and by oceanic crust, $U_{\mathrm{pe}, \mathrm{o}, \mathrm{l}}$. These contributions are given by

$U_{\mathrm{pe}, \mathrm{c}, 1}=\int_{0}^{\Delta z_{\mathrm{c}, 1}} L_{\mathrm{c}} \rho_{\mathrm{c}} g z \mathrm{~d} z=\frac{L_{\mathrm{c}} \rho_{\mathrm{c}} g}{2} \Delta z_{\mathrm{c}, \mathrm{l}}^{2}$ 
and

$U_{\mathrm{pe}, \mathrm{o}, \mathrm{l}}=\int_{0}^{\Delta z_{\mathrm{o}, 1}}\left(L-L_{\mathrm{c}}\right) \rho_{\mathrm{o}} g z \mathrm{~d} z=\frac{\left(L-L_{\mathrm{c}}\right) \rho_{\mathrm{o}} g}{2} \Delta z_{\mathrm{o}, \mathrm{l}}^{2}$.

Using Eqs. (A2) and (A4) to express $\Delta z_{\mathrm{c}, 1}$ and $\Delta z_{\mathrm{o}, 1}$ in terms of the masses $m_{\mathrm{c}}$ and $m_{\mathrm{o}}$, the total potential energy is expressed by

$U_{\mathrm{pe}, \mathrm{tot}, \mathrm{l}}=U_{\mathrm{pe}, \mathrm{o}, \mathrm{l}}+U_{\mathrm{pe}, \mathrm{c}, \mathrm{l}}=\frac{g}{2 L_{\mathrm{c}} \rho_{\mathrm{c}}} m_{\mathrm{c}}^{2}+\frac{g}{2\left(L-L_{\mathrm{c}}\right) \rho_{\mathrm{o}}} m_{\mathrm{o}}^{2}$.

\section{A1.3 Potential energy in global, stratigraphic equilibrium}

The potential energy of the configuration shown in Fig. 1c is derived equivalently. The individual contributions by the continental and oceanic crust are given by

$$
U_{\mathrm{pe}, \mathrm{c}, \mathrm{g}}=\int_{0}^{\Delta z_{\mathrm{c}, \mathrm{g}}} L \rho_{\mathrm{c}} g z \mathrm{~d} z=\frac{L \rho_{\mathrm{c}} g}{2}\left(\Delta z_{\mathrm{c}, \mathrm{g}}^{2}-\Delta z_{\mathrm{o}, \mathrm{g}}^{2}\right)
$$

and

$U_{\mathrm{pe}, \mathrm{o}, \mathrm{g}}=\int_{0}^{\Delta z_{\mathrm{o}, \mathrm{g}}} L \rho_{\mathrm{o}} g z \mathrm{~d} z=\frac{L \rho_{\mathrm{o}} g}{2} \Delta z_{\mathrm{o}, \mathrm{g}}^{2}$.

Taken together, and using Eqs. (A6) and (A8) as above to express $\Delta z_{\mathrm{c}, \mathrm{g}}$ and $\Delta z_{\mathrm{o}, \mathrm{g}}$ in terms of the masses $m_{\mathrm{c}}$ and $m_{\mathrm{o}}$, we obtain

$$
U_{\mathrm{pe}, \mathrm{tot}, \mathrm{g}}=U_{\mathrm{pe}, \mathrm{o}, \mathrm{g}}+U_{\mathrm{pe}, \mathrm{c}, \mathrm{g}}=\frac{g}{2 L \rho_{\mathrm{c}}} m_{\mathrm{c}}^{2}+\frac{g}{2 L \rho_{\mathrm{o}}} m_{\mathrm{o}}^{2}\left(1-\frac{\rho_{\mathrm{c}}}{\rho_{\mathrm{o}}}\right) .
$$

\section{A1.4 Difference in potential energy}

We now consider the difference in potential energy, $\Delta U_{\text {pe,tot }}$, between the global equilibrium state shown in Fig. 1c to the local equilibrium state shown in Fig.1a. We use Eqs. (A11) and (A14) to get the following expression for $\Delta U_{\mathrm{pe}, \text { tot }}$ :

$$
\begin{aligned}
& \Delta U_{\text {pe }, \text { tot }}=U_{\mathrm{pe}, \mathrm{tot}, \mathrm{g}}-U_{\mathrm{pe}, \mathrm{tot}, \mathrm{l}}=\frac{g m_{\mathrm{c}}^{2}}{2 \rho_{\mathrm{c}}}\left(\frac{1}{L}-\frac{1}{L_{\mathrm{c}}}\right) \\
& +\frac{g m_{\mathrm{o}}^{2}}{2 \rho_{\mathrm{o}}}\left(\frac{1}{L}\left(1-\frac{\rho_{\mathrm{c}}}{\rho_{\mathrm{o}}}\right)-\frac{1}{L-L_{\mathrm{c}}}\right) \\
& =-\frac{g m_{\mathrm{c}}^{2}}{2 \rho_{\mathrm{c}} L_{\mathrm{c}}}\left(1-\frac{L_{\mathrm{c}}}{L}\right) \\
& -\frac{g m_{\mathrm{o}}^{2}}{2 \rho_{\mathrm{o}}\left(L-L_{\mathrm{c}}\right)}\left(1-\left(1-\frac{L_{\mathrm{c}}}{L}\right)\left(1-\frac{\rho_{\mathrm{c}}}{\rho_{\mathrm{o}}}\right)\right) .
\end{aligned}
$$

What can be seen from Eq. (A15) is that both terms are negative, i.e. that the potential energy decreases from the state shown in Fig 1a to c.

\section{A1.5 Summary}

In summary, this note shows in relatively simple terms that the transition from a local isostatic equilibrium to a global stratigraphic equilibrium is accompanied by a reduction of potential energy in the overall system.

In principle, one could also show that the initial state of local isostatic equilibrium represents a state of minimum potential energy with respect to the vertical position of continental crust, $\Delta z_{\mathrm{c}, \mathrm{j}}$, and that the state of global equilibrium represents a state of minimum potential energy with respect to the horizontal extent of continental crust, $L_{\mathrm{c}}$. This would, however, require quite lengthly algebraic computations, which has been omitted here for reasons of brevity.

Acknowledgements. We thank one anonymous reviewer, $\mathrm{Hu}-$ bert Savenije, Kyungrock Paik, and Greg Tucker for very constructive reviews that helped to improve this manuscript. A. K. acknowledges financial support from the Helmholtz Alliance "Planetary Evolution and Life". This research contributes to the "Catchments As Organized Systems (CAOS)" research group funded by the German Science Foundation (DFG).

The service charges for this open access publication have been covered by the Max Planck Society.

Edited by: M. Sivapalan

\section{References}

Ahnert, F.: Functional relationships between denudation, relief and uplift in large mid-latitude drainage basins, Am. J. Sci., 268, 243-263, 1970.

Bagnold, R. A.: An approach to the sediment transport problem from general physics, US Geological Survey professional paper 422-I, US government printing officce, Washington, DC, USA, 1966.

Bejan, A.: Advanced Engineering Thermodynamics, Wiley, New York, 1997.

Bejan, A. and Lorente, S.: The constructal law and the evolution of design in nature, Phys. Life Rev., 8, 209-240, 2011.

Dewar, R. C.: Maximum entropy production and the fluctuation theorem, J. Phys. A, 38, 371-381, 2005.

Dewar, R. C.: Maximum entropy production as an inference algorithm that translates physical assumptions into macroscopic predictions: don't shoot the messenger, Entropy, 11, 931-944, 2010.

Dietrich, W. E. and Perron, J. T.: The search for a topographic signature of life, Nature, 439, 411-418, 2006.

Dyke, J. G., Gans, F., and Kleidon, A.: Towards understanding how surface life can affect interior geological processes: a nonequilibrium thermodynamics approach, Earth Syst. Dynam., 2, 139-160, doi:10.5194/esd-2-139-2011, 2011.

Howard, A. D.: Theoretical model of optimal drainage networks, Water Resour. Res., 26, 2107-2117, 1990.

Izumi, N. and Parker, G.: Linear stability analysis of channel inception: downstream-driven theory, J. Fluid Mech., 419, 239-262, 2000 . 
Kirkby, M. J.: Hillslope process-response models based on the continuity equation, Inst. Brit. Geogr., Spec. Publ., 3, 15-30, 1971.

Kleidon, A.: Life, hierarchy, and the thermodynamic machinery of planet Earth, Phys. Life Rev., 7, 424-460, 2010a.

Kleidon, A.: A basic introduction to the thermodynamics of the Earth system far from equilibrium and maximum entropy production, Philos. T. Roy. Soc. B, 365, 1303-1315, 2010 b.

Kleidon, A.: How does the earth system generate and maintain thermodynamic disequilibrium and what does it imply for the future of the planet?, Philos. T. Roy. Soc. A, 370, 1012-1040, 2012.

Kleidon, A. and Schymanski, S. J.: Thermodynamics and optimality of the water budget on land: a review, Geophys. Res. Lett., 35, L20404, doi:10.1029/2008GL035393, 2008.

Kleidon, A., Malhi, Y., and Cox, P. M.: Maximum entropy production in environmental and ecological systems, Philos. T. Roy. Soc. B, 365, 1297-1302, 2010.

Kleidon, A., Zehe, E., and Lin, H. S.: Thermodynamic Limits in the Critical Zone and Its Relevance to Hydropedology, in: Hydropedology: Synergistic Integration of Soil Science and Hydrology, edited by: Lin, H., Elsevier, Amsterdam, The Netherlands, $243-$ 284, 2012a.

Kleidon, A., Zehe, E., Ehret, U., and Scherer, U.: Interactive Comment on "Thermodynamics, maximum power, and the dynamics of preferential river flow structures on continents" by A. Kleidon et al., Hydrol. Earth Syst. Sci. Discuss., 9, C3466-C3469, 2012 b.

Lenton, T. M.: Gaia and natural selection, Nature, 394, 439-447, 1998.

Leopold, L. B. and Langbein, W. B.: The concept of entropy in landscape evolution, US Geol. Surv. Prof. Pap. 500-A, US government printing office, Washington, DC, USA, 1962.

Lotka, A. J.: Contribution to the energetics of evolution, P. Natl. Acad. Sci. USA, 8, 147-151, 1922a.

Lotka, A. J.: Natural selection as a physical principle, P. Natl. Acad. Sci. USA, 8, 151-154, 1922b.

Odum, E. P.: The strategy of ecosystem development, Science, 164, 262-270, 1969.

Odum, H. T.: Self-organization, transformity, and information, Science, 242, 1132-1139, 1988.

Ozawa, H., Ohmura, A., Lorenz, R. D., and Pujol, T.: The second law of thermodynamics and the global climate system: a review of the maximum entropy production principle, Rev. Geophys., 41, 1018, doi:10.1029/2002RG000113, 2003.

Paik, K.: Optimization approach for 4-D natural landscape evolution, IEEE T. Evol. Comput., 15, 684-691, 2011.

Paik, K. and Kumar, P.: Optimality approaches to describe characteristic fluvial patterns on landscapes, Philos. T. Roy. Soc. B, 365, 1387-1395, 2010.
Paltridge, G. W.: Global dynamics and climate - a system of minimum entropy exchange, Q. J. Roy. Meteorol. Soc., 101, 475-484, 1975.

Paltridge, G. W.: Climate and thermodynamic systems of maximum dissipation, Nature, 279, 630-631, 1979.

Phillips, J. D.: Biological energy in landscape evolution, Am. J. Sci., 309, 271-289, doi:10.2475/04.2009.01, 2009.

Phillips, J. D.: The job of the river, Earth Surf. Proc. Land., 35, 305-313, 2010.

Rinaldo, A., Rodriguez-Iturbe, I., Rigon, R., Bras, R. L., IjjaszVasquez, E., and Marani, A.: Minimum energy and fractal structures of drainage networks, Water Resour. Res., 28, 2183-2195, 1992.

Rodriguez-Iturbe, I. and Rinaldo, A.: Fractal River Basins, Chance and Self-Organization, Cambridge University Press, New York, 1997.

Rodriguez-Iturbe, I., Rinaldo, A., Rigon, R., Bras, R. L., Marani, A., and Ijjasz-Vasquez, E.: Energy dissipation, runoff production, and the three-dimensional structure of river basins, Water Resour. Res., 28, 1095-1103, 1992a.

Rodriguez-Iturbe, I., Rinaldo, A., Rigon, R., Bras, R. L., IjjaszVasquez, E., and Marani, A.: Fractal structures as least energy patterns: the case of river networks, Geophys. Res. Lett., 9, 889892, 1992b.

Smith, T. R.: A theory for the emergence of channelized drainage, J. Geophys. Res., 115, F02023, doi:10.1029/2008JF001114, 2010.

Smith, T. R. and Bretherton, F. P.: Stability and the conservation of mass in drainage basin evolution, Water Resour. Res., 8, 15061529, 1972.

Wang, J. and Bras, R. L.: A model of evapotranspiration based on the theory of maximum entropy production, Water Resour. Res., 47, W03521, doi:10.1029/2010WR009392, 2011.

West, G. B., Brown, J. H., and Enquist, B. J.: A general model for the origin of allometric scaling laws in biology, Science, 276, 122-126, 1997.

Whipple, K. X. and Tucker, G. E.: Implications of sediment-fluxdependent river incision models for landscape evolution, J. Geophys. Res., 107, 2039, doi:10.1029/2000JB000044, 2002.

Woldenberg, M. J.: Spatial order in fluvial systems: Horton's laws derived from mixed hexagonal hierarchies of drainage basin areas, Geol. Soc. Am. Bull., 80, 97-112, 1969.

Zehe, E., Blume, T., and Blöschl, G.: The principle of maximum energy dissipation: a novel thermodynamic perspective on rapid water flow in connected soil structures, Philos. T. Roy. Soc. B, 365, 1377-1386, 2010. 